\title{
Stochastic competitive exclusion leads to a cascade of species extinctions
}

\author{
José A. Capitán ${ }^{\mathrm{a}, \mathrm{c}, *}$, Sara Cuenda ${ }^{\mathrm{b}}$, David Alonso ${ }^{\mathrm{c}}$ \\ a Department of Applied Mathematics, Technical University of Madrid, Madrid, Spain

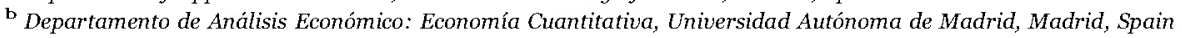 \\ c Center for Advanced Studies (CEAB-CSIC), Blanes, Catalunya, Spain
}

\section{A R T I C L E I N F O}

Keywords:

Competitive exclusion

Ecological drift

Continuous-time Markov processes

\begin{abstract}
A B S T R A C T
Community ecology has traditionally relied on the competitive exclusion principle, a piece of common wisdom in conceptual frameworks developed to describe species assemblages. Key concepts in community ecology, such as limiting similarity and niche partitioning, are based on competitive exclusion. However, this classical paradigm in ecology relies on implications derived from simple, deterministic models. Here we show how the predictions of a symmetric, deterministic model about the way extinctions proceed can be utterly different from the results derived from the same model when ecological drift (demographic stochasticity) is explicitly considered. Using analytical approximations to the steady-state conditional probabilities for assemblages with two and three species, we demonstrate that stochastic competitive exclusion leads to a cascade of extinctions, whereas the symmetric, deterministic model predicts a multiple collapse of species. To test the robustness of our results, we have studied the effect of environmental stochasticity and relaxed the species symmetry assumption. Our conclusions highlight the crucial role of stochasticity when deriving reliable theoretical predictions for species community assembly.
\end{abstract}

\section{Introduction}

Ecological communities are shaped from the complex interplay of four fundamental processes (Vellend, 2010): selection, in the form of species interactions that favor certain species against others; speciation, leading to the appearance of new species, better adapted to the environment; dispersal, which permits spatial propagation of individuals; and ecological drift, a demographic variability in species population numbers due to the stochastic processes that take place. Ecological drift, in particular, has a prevalent role in modern theoretical frameworks in community ecology (Black and McKane, 2012). Accordingly, current approaches reveal the need for process-based, stochastic models that help to understand how ecological communities are assembled and their interaction with environmental factors (Wisz et al., 2013).

Classical community ecology, however, has mainly relied on deterministic community models (see Roughgarden, 1979 and references therein), most of them based on Lotka-Volterra dynamics, although alternatives have been proposed (Schoener, 1974a). There is a long-standing research focus on community assembly models, in which communities are built up through species invasions, and most of them rely on deterministic approaches (Post and Pimm, 1983; Law and Morton, 1993, 1996; Capitán et al., 2009; Capitán and Cuesta, 2011;
Capitán, 2011). On the other side, there have been strong theoretical efforts to describe community assemblages in stochastic terms (Hubbell, 2001; Alonso et al., 2008; Rosindell et al., 2011). In certain situations, the results and conclusions derived from deterministic models have been shown to be quite different in the presence of stochasticity (Bolker and Grenfell, 1995; Alonso et al., 2007; Haegeman and Loreau, 2011; Bonachela et al., 2012; Wang et al., 2012).

One of the contexts where the differences between deterministic and stochastic approaches become apparent is related to theoretical formulations of the competitive exclusion principle (Volterra, 1926; Gause, 1934; Hardin, 1960). This principle constitutes a fundamental pillar of community ecology and belongs to the traditional body of ecological theory. It provides a useful theoretical framework to explore how complex species assemblages persist over time. Important concepts such as adaptation to shared niches (Roughgarden, 1979), species limiting similarity (MacArthur and Levins, 1967; Roughgarden, 1974) or niche partitioning (Pielou, 1977; Schoener, 1974b) all are immediate derivations from the principle. Classical approaches predict the maximum degree of species similarity that permit species stable coexistence (MacArthur, 1969, 1970). However, theoretical predictions for limiting similarity often rely on deterministic community models (see MacArthur, 1968; Levin, 1970; Haigh and

\footnotetext{
* Corresponding author at: Department of Applied Mathematics, Technical University of Madrid, Madrid, Spain.

E-mail addresses: ja.capitan@upm.es (J.A. Capitán), sara.cuenda@uam.es (S. Cuenda), dalonso@iceab.csic.es (D. Alonso).
} 
Maynard-Smith, 1972; Chesson, 1990 and Appendix A for a discussion on competitive exclusion based on deterministic approaches), and the relevance of stochasticity, in the form of ecological drift, to species coexistence has remained almost unexplored (with the exception of Turelli (1980)). The relationship between limiting similarity and environmental stochasticity has been studied more thoroughly (May and MacArthur, 1972; Turelli, 1978, 1981).

Recently, we focused on the influence of ecological drift on the similarity of coexisting species via the competitive exclusion principle (Capitán et al., 2015). In that contribution we showed that, in the presence of ecological drift, the maximum degree of similarity that ensures stable coexistence can be significantly lowered when compared to the corresponding limits to similarity derived from deterministic models. If similarity is interpreted in terms of an interspecific competitive overlap (MacArthur and Levins, 1967; Roughgarden, 1974), stochasticity displaces the deterministic threshold towards lower values of the competitive overlap (Capitán et al., 2015). Thus, when stochasticity is considered, the extinction phenomena caused by competitive exclusion takes place at lower values of the competitive overlap (i.e., species have to be more dissimilar to stably coexist in the presence of ecological drift).

Ecological drift becomes a key process determining species coexistence in aspects other than the maximum similarity of co-occurring species. Beyond a more restrictive threshold in competition induced by ecological drift (which was the main result of Capitán et al. (2015)), we here analyze the influence of demographic stochasticity on the extinction mechanism itself, which in principle can lead to either sequential or grouped extinctions as competition strength increases. For that purpose, we considered a deterministic, Lotka-Volterra model and its stochastic counterpart, both of which treat species interactions symmetrically. Whereas the deterministic model predicts the multiple extinction of all the species in the community but one as competition crosses over a certain threshold, in the presence of demographic stochasticity extinctions proceed progressively, in the form of a cascade, as competition increases. The only difference between both approaches is the explicit consideration of ecological drift in the dynamics. In order to derive our conclusions, we developed convenient analytical approximations to the steady-state configurations of the stochastic system for simple species assemblages formed by two or three species. Such approximations help us to partition the set of feasible population numbers into regions associated to coexistence, or the extinction of one, two, or three species. The steady-state probabilities, when aggregated over those regions, unveil the extinction cascade phenomenon. Our main result reveals overlapping windows in competitive strength, at low values related to configurations where the coexistence of three species is the most probable state, intermediate ranges where it is more likely to observe two-species assemblages, and large competition values for which the most probable state is formed by one species or none. We also studied the transition to the deterministic model when demographic stochasticity tends to zero, and our results reveal an abrupt transition to situations compatible with small stochasticity.

To test the robustness of our conclusions, we replaced demographic stochasticity by environmental stochasticity and confirmed that, although the extinction phenomena are qualitatively different, the extinction cascade persists. We also relaxed the assumption of symmetry to assess the effect of stochasticity on deterministic models that not only predict multiple extinctions, as in the fully symmetric scenario, but also lead to both progressive and grouped extinctions for fixed competitive strengths. When stochasticity comes into play, however, the stochastic cascade persists and the expected extinction sequence is qualitatively different from its deterministic counterpart. Thus, the predictions of both models are significantly different in generic, non-symmetric scenarios for species interactions.

The paper is organized as follows: in Section 2 we describe both the deterministic and the stochastic frameworks, the latter based on the formulation of Haegeman and Loreau (2011), and show that the deterministic, symmetric model predicts a multiple species extinction. In Section 3 we start by presenting the analytical approximations for a two-species stochastic community model, and we then extend the procedure to a three-species community. These approximations help us to obtain analytical formulae for the critical points of the steady-state, joint probability distribution of the community. Formulae for saddle points are then used to properly define aggregated probabilities of coexistence, or one-, two-, and three-species extinction, which reveal themselves the sequential decline of species driven by ecological drift. After studying the small stochasticity limit and testing the robustness of our results, we conclude the paper with several implications and prospects (Section 4).

\section{Model description}

For the sake of simplicity, in this contribution we will focus on the symmetric version of the deterministic Lotka-Volterra competitive dynamics (see Appendix A),

$\dot{x}_{i}=\pi x_{j}\left(1-\frac{x_{i}+\beta \sum_{j \neq i} x_{j}}{K}\right), \quad i=1, \ldots, S$,

where $x_{i}$ stands for the population density of species $i$ (space is implicitly assumed) and model parameters are uniform and speciesindependent. Here $r$ stands for an intrinsic, species-independent growth rate, $\rho$ measures interspecific competition, $K$ represents a carrying capacity, and $S$ is the species richness of the community. The dynamies has an interior equilibrium point, $\widehat{\mathbf{x}}=(\widehat{x}, \ldots, \widehat{x})$, where $\hat{x}=K /(1-\rho+\rho S)$, which is globally stable if and only if $\rho<1$ (Hofbauer and Sigmund, 1998; Capitán et al., 2015). In the symmetric scenario, the competitive exclusion principle adopts a very simple formulation (see Appendix A for further details on the general, nonsymmetric case). A complete stability analysis of the boundary equilibrium points shows that, for $p>1$, all the species become extinct except for just one of them (see Appendix B). As a consequence, competitive exclusion in the symmetric, deterministic model implies the joint extinction of $S-1$ species.

We now explicitly incorporate ecological drift (demographic stochasticity) in the symmetric scenario in order to show that species are sequentially displaced in the presence of stochasticity due to competitive exclusion, following a cascade of extinctions, as competition strengthens. A standard way to extend deterministic models to incorporate ecological drift is deeply described in Haegeman and Loreau (2011). The state of the system is described by the vector of population numbers $n_{i}$ at time $t, \mathbf{n}(t)=\left(n_{1}(t) \ldots, n_{S}(t)\right)$. Contrary to the deterministic case, which focuses on population densities $x_{i}=n_{i} / \Omega, \Omega$ being a meaningful measure (area, volume) of the system size, here discrete population numbers are considered. The elementary processes that define the stochastic dynamics (local births and deaths, immigration, and competition) are characterized by probability rates that, in the deterministic limit, yield the Lotka-Volterra Eq. (1). As in Haegeman and Loreau (2011), we choose the following probability rates to model elementary processes:

1. Local births (deaths) of species $i$ occur at a density-independent rate $r^{+} n_{i}\left(r^{-} n_{i}\right)$. We adopt the notation $r=F^{+}-r^{-}$to represent the net growth rate in the absence of competitors.

2. Immigration of a new individual of species $i$ takes place at a rate $\mu$. Although the deterministic model (1) does not include immigration, dispersal is an important process driving community assembly (Vellend, 2010). In addition, immigration is key for the stochastic process to reach a non-trivial steady-state. We consider here the lowimmigration regime, in which the deterministic limit is expected to recover results close to those yielded by Eq. (1), see Capitán et al. (2015). 
3. Intraspecific competition occurs at a density-dependent rate $m_{i}^{2} / K$, where $K$ represents a species carrying capacity.

4. Interspecific competition between species $i$ and $j(i \neq j)$ takes place at a probability $r \rho n_{i} n_{j} / K$ per unit time (it is also a density-dependent rate).

Population vectors $\mathbf{n}(t)$ belong to the configuration space $\mathbb{N}^{s}$, where $\mathbb{N}=\{0,1,2, \ldots\}$. The elementary processes listed above define a birthdeath-immigration stochastic process in continuous time, and the probability $P(\mathbf{n}, t)$ of finding a population vector $\mathbf{n}(t)$ at time $t$ is determined by the master equation,

$$
\begin{aligned}
\frac{\partial P(\mathbf{n}, t)}{\partial t}= & \sum_{i=1}^{S}\left\{q_{i}^{+}\left(\mathbf{n}-\mathbf{e}_{i}\right) P\left(\mathbf{n}-\mathbf{e}_{i}, t\right)+q_{i}^{-}\left(\mathbf{n}+\mathbf{e}_{i}\right) P\left(\mathbf{n}+\mathbf{e}_{i}, t\right)\right. \\
& \left.-\left[q_{i}^{+}(\mathbf{n})+q_{i}^{-}(\mathbf{n})\right] P(\mathbf{n}, t)\right\} .
\end{aligned}
$$

Here $q_{i}^{+}(\mathbf{n})=r^{+} n_{i}+\mu$ is the overall birth rate for species $i$, $q_{i}^{-}(\mathbf{n})=r^{-} n_{i}+r n_{i}\left(n_{i}+\rho \sum_{j \neq i} n_{j}\right) / K$ is the overall death rate, and $\mathbf{e}_{i}=(0, \ldots, 1, \ldots, 0)$ is the $i$-th vector of the canonical basis of $\mathbb{R}^{S}$. Notice the correspondence of these rates with the terms arising in the deterministic model (1) for $\mu=0$. The steady-state probability distribution is obtained by solving the coupled recurrence equation given by the condition $\partial P(\mathbf{n}, t) / \partial t=0$ (see Appendix C for details).

Our approach develops analytical approximations for the critical points of the joint probability function, not for the probability itself. For $S=1$ the stationary state of the birth-death-immigration model can he exactly solved in terms of hypergeometric functions (Haegeman and Loreau, 2011). For $S>1$, in the absence of competition $(\rho=0)$ populations are uncorrelated, and the joint probability distribution factors as a product of marginal probabilities, which reduces this case to a one-dimensional problem. The fully neutral model $(\rho=1)$ for $S>1$ can be solved as well (Haegeman and Loreau, 2011). We cannot find analytically the steady-state distribution for $S \geq 2$ and $\rho>0$, though. Haegeman and Loreau (2011) devised approximations for this case, but we will follow here a different approach to find analytical formulae for the critical points of the steady-state distribution in the case of small-sized communities.

\section{Results}

In Capitán et al. (2015) we showed numerically that the steadystate distribution for two-species communities presents a maximum at an interior point of the configuration space, as well as two boundary maxima with population vectors of the form $(n, 0)$ and $(0, n)$. This implies that the discrete probability distribution, when extended to be real-valued (by, for instance, cubic spline interpolation), must exhibit by continuity two saddle points located in between the coexistence maximum and the two boundary maxima. These saddle points can be used to conveniently partition the configuration space into regions associated to coexistence, the extinction of one species, or the extinction of two species. In this section we develop analytical approximations that help us to obtain good estimates for the critical points of the joint probability distribution. We use the $S=2$ case to illustrate the technique. We then extend the method for three-species assemblages, and use the approximated saddle points to partition the configuration space into regions for coexistence and for the three possible states where extinctions have occurred. The aggregation of the joint probability over those regions unveils the extinction cascade phenomenon.

\subsection{Critical points for two-species communities}

To estimate the location of the critical points of the joint distribution $P\left(n_{1}, n_{2}\right)$ we use that the conditions $\partial P\left(n_{1}, n_{2}\right) / \partial n_{1}=0$ and $\partial P\left(n_{1}, n_{2}\right) / \partial n_{2}=0$ are equivalent to $\frac{\partial}{\partial n_{1}} P\left(n_{1} \mid n_{2}\right)=\frac{\partial}{\partial n_{1}}\left(\frac{P\left(n_{1}, n_{2}\right)}{P\left(n_{2}\right)}\right)=0$

$\frac{\partial}{\partial n_{2}} P\left(n_{2} \mid n_{1}\right)=\frac{\partial}{\partial n_{2}}\left(\frac{P\left(n_{1}, n_{2}\right)}{P\left(n_{1}\right)}\right)=0$,

$P\left(n_{1} \mid n_{2}\right)$ being the probability that species 1 has $n_{1}$ individuals conditioned to species 2 having $n_{2}$ individuals. This means that critical points of the joint distribution $P\left(n_{1}, n_{2}\right)$ can also be obtained through conditional probabilities. By fixing $n_{2}$, we just need to evaluate the derivative of $P\left(n_{1} \mid n_{2}\right)$ along the $n_{1}$ direction. The same applies under the change $n_{1} \leftrightarrow n_{2}$.

\subsubsection{Approximated conditional probabilities}

We now approximate $P\left(n_{2} \mid n_{1}\right)$ by $T\left(n_{2} \mid n_{1}\right)$ as follows. For $S=2$, the steady-state distribution satisfies a two-term recurrence in population numbers $n_{1}$ and $n_{2}$, namely

$$
\begin{aligned}
0= & q_{1}^{+}\left(n_{1}-1, n_{2}\right) P\left(n_{1}-1, n_{2}\right)+q_{1}^{-}\left(n_{1}+1, n_{2}\right) P\left(n_{1}+1, n_{2}\right) \\
& +q_{2}^{+}\left(n_{1}, n_{2}-1\right) P\left(n_{1}, n_{2}-1\right)+q_{2}^{-}\left(n_{1}, n_{2}+1\right) P\left(n_{1}, n_{2}+1\right) \\
& -\left[q_{1}^{+}\left(n_{1}, n_{2}\right)+q_{1}^{-}\left(n_{1}, n_{2}\right)\right] P\left(n_{1}, n_{2}\right)-\left[q_{2}^{+}\left(n_{1}, n_{2}\right)+q_{2}^{-}\left(n_{1}, n_{2}\right)\right] P\left(n_{1}, n_{2}\right) .
\end{aligned}
$$

Notice that we are interested in approximating the conditional probability $P\left(n_{2} \mid n_{1}\right)$ where $n_{1}$ is fixed. Hence, in the approximation we ignore the terms in Eq. (4) that involve variation of $n_{1}$, and assume that $n_{1}$ acts as a fixed parameter in the remaining terms. Thus, the approximated conditional probability $T\left(n_{2} \mid n_{1}\right)$ satisfies

$$
\begin{aligned}
0= & q_{2}^{+}\left(n_{1}, n_{2}-1\right) T\left(n_{2}-1 \mid n_{1}\right)+q_{2}^{-}\left(n_{1}, n_{2}+1\right) T\left(n_{2}+1 \mid n_{1}\right) \\
& -\left[q_{2}^{+}\left(n_{1}, n_{2}\right)+q_{2}^{-}\left(n_{1}, n_{2}\right)\right] T\left(n_{2} \mid n_{1}\right) .
\end{aligned}
$$

This expression fulfills a detailed balance condition (Karlin and Taylor, 1975), which yields an approximate one-term recurrence formula in $n_{2}$,

$$
\begin{aligned}
T\left(n_{2} \mid n_{1}\right) & =\frac{q_{2}^{+}\left(n_{1}, n_{2}-1\right)}{q_{2}^{-}\left(n_{1}, n_{2}\right)} T\left(n_{2}-1 \mid n_{1}\right) \\
& =\frac{K\left[\mu+r^{+}\left(n_{2}-1\right)\right]}{n_{2}\left[K r^{-}+r\left(n_{2}+\rho n_{1}\right)\right]} T\left(n_{2}-1 \mid n_{1}\right),
\end{aligned}
$$

and leads to an explicit solution in terms of hypergeometric functions, as in Haegeman and Loreau (2011). A symmetric recurrence holds for $T\left(n_{1} \mid n_{2}\right)$.

\subsubsection{Analytical formulae for critical points}

Our next step is to approximate the partial derivative along the $n_{1}$ direction by the backwards discrete difference (comparable results are obtained with the forward difference),

$\frac{\partial}{\partial n_{1}} P\left(n_{1} \mid n_{2}\right) \approx \frac{\partial}{\partial n_{1}} T\left(n_{1} \mid n_{2}\right) \approx T\left(n_{1} \mid n_{2}\right)-T\left(n_{1}-1 \mid n_{2}\right)$.

Similarly,

$\frac{\partial}{\partial n_{2}} P\left(n_{2} \mid n_{1}\right) \approx T\left(n_{2} \mid n_{1}\right)-T\left(n_{2}-1 \mid n_{1}\right)$.

Eq. (6) allows us to write the system for the critical points of the twodimensional joint-probability surface, $\partial P\left(n_{1} \mid n_{2}\right) / \partial n_{1}=\partial P\left(n_{2} \mid n_{1}\right) / \partial n_{2}=0$, as

$n_{1}^{2}+\rho n_{1} n_{2}-K n_{1}+K \alpha=0$,

$n_{2}^{2}+\rho n_{1} n_{2}-K n_{2}+K \alpha=0$,

where $\alpha=\left(r^{+}-\mu\right) / r$. Solving the quadratic system yields the following estimates for the interior critical points: $M_{1}=\left(m_{+}, m_{+}\right)$, which can be either a local maximum or a saddle point depending on the value of $\rho$ (see below), and the local minimum $M_{2}=\left(m_{-}, m_{-}\right)$, where 

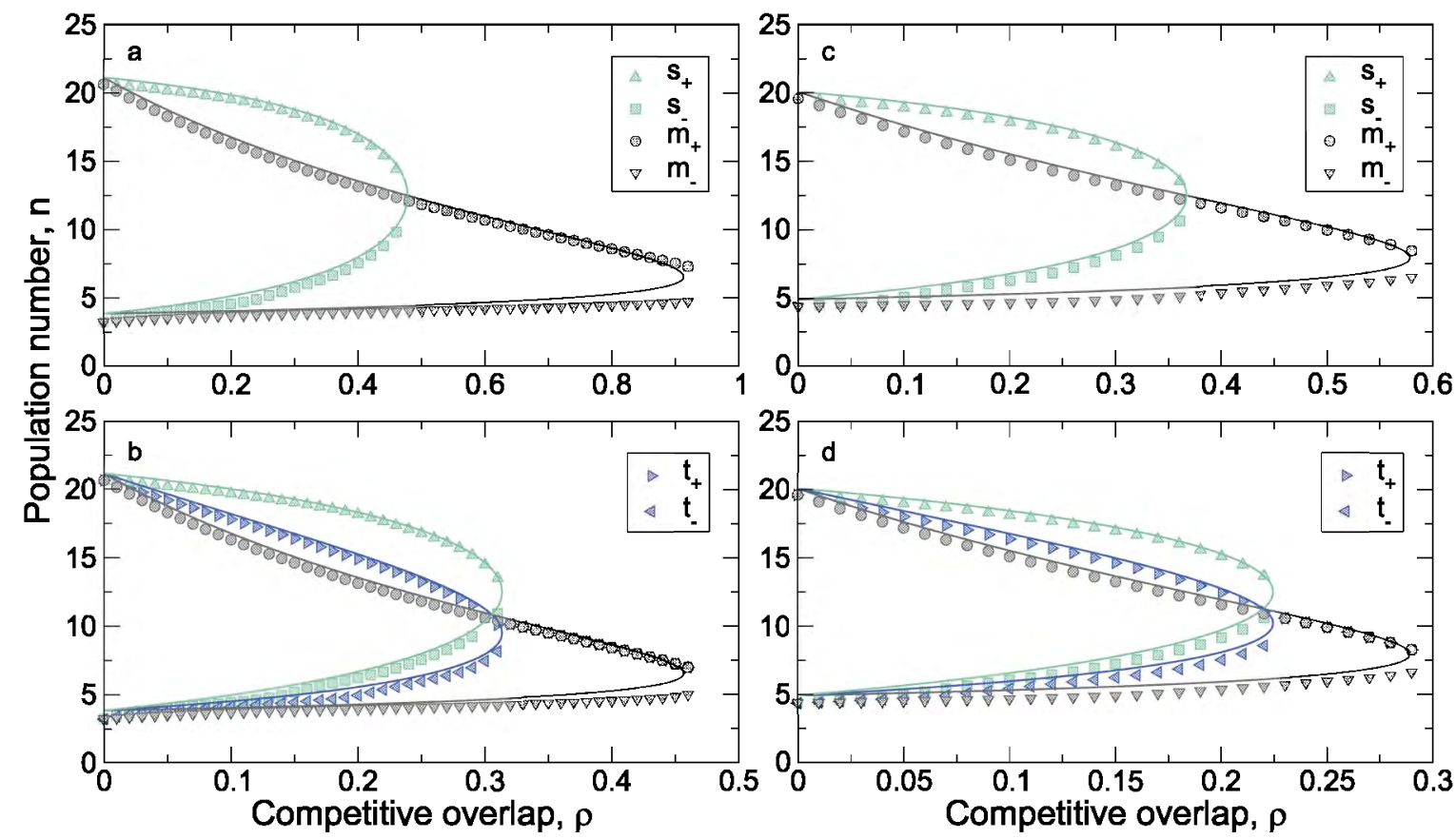

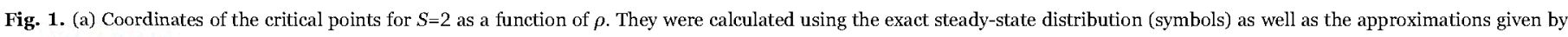

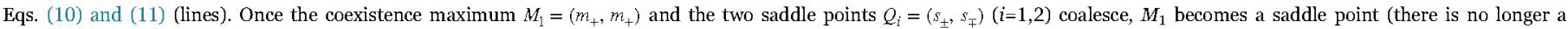

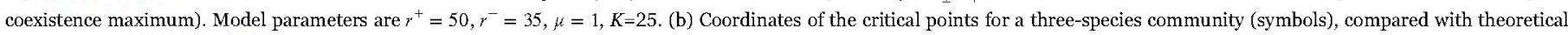

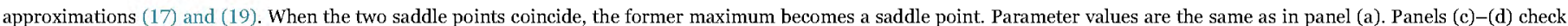

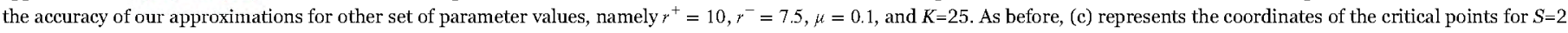
and (d) for $S=3$.

$m_{ \pm}=\frac{K}{2(1+\rho)}\left(1 \pm \sqrt{1-\frac{4 \alpha(1+\rho)}{K}}\right)$,

and two (symmetrical) saddle points $Q_{1}=\left(s_{+}, s_{-}\right)$and $Q_{2}=\left(s_{-}, s_{+}\right)$, where

$s_{ \pm}=\frac{K}{2}\left(1 \pm \sqrt{1-\frac{4 \alpha}{K(1-\rho)}}\right)$

To test the goodness of these analytical approximations, critical points of the exact probability distribution are determined numerically by extending the discrete distribution $P\left(n_{1}, n_{2}\right)$ to be a real-valued function using cubic spline interpolation both in the $n_{1}$ and $n_{2}$ directions. We then solve numerically the system $\partial P\left(n_{1}, n_{2}\right) / \partial n_{1}=\partial P\left(n_{1}, n_{2}\right) / \partial n_{2}=0$, using analytical predictions as initial guesses for iterative root finding. The Hessian matrix decides whether a given point is a local maximum, a local minimum or a saddle point. We find that the critical points are in excellent agreement with the analytical formulae above (see Fig. 1a, in which we plot the coordinates of each critical point calculated both analytically and numerically).

For $\rho<\rho_{c}$, with

$\rho_{c}=1-\frac{4 \alpha}{K}=1-\frac{4\left(r^{+}-\mu\right)}{K r}$,

the analysis of the Hessian matrix reveals that $M_{1}$ is a (coexistence) local maximum. For $\rho=\rho_{c}$, saddle points $Q_{1}$ and $Q_{2}$ coincide with $M_{1}$ and, for $\rho>\rho_{c}, M_{1}$ transforms into a saddle point and the former saddle points $Q_{1}$ and $Q_{2}$ (cf. Eq. (11)) no longer exist. Moreover, when $\rho>K / 4 \alpha-1$, all interior critical points (10) become complex and the only persistent maxima are those located at the boundary.

There are four critical points at the boundary, which can be approximated using Eq. (6) for $n_{1}=0$, resulting in two local maxima $\left[\left(b_{+}, 0\right),\left(0, b_{+}\right)\right]$and two local minima $\left[\left(b_{-}, 0\right),\left(0, b_{-}\right)\right]$, where

$b_{ \pm}=\frac{K}{2}\left(1 \pm \sqrt{1-\frac{4 \alpha}{K}}\right)$.
Observe that the non-zero coordinates of the local maxima (minima) coincide with those of the interior maximum (minimum) for $\rho=0$.

\subsection{Critical points for three-species communities}

The method proposed in the previous subsection can be fully extended to the case of a three-species community. Critical points are obtained by approximating conditional probabilities $P\left(n_{1} \mid n_{2}, n_{3}\right)$ and taking discrete derivatives with respect to the first argument. As before, we consider the three-term recurrence relation that fulfills the joint distribution $P\left(n_{1}, n_{2}, n_{3}\right)$ and ignore the terms that involve variation in population numbers $n_{2}$ and $n_{3}$. Under this approximation, the steadystate condition reduces to

$$
\begin{aligned}
0= & q_{1}^{+}\left(n_{1}-1, n_{2}, n_{3}\right) T\left(n_{1}-1 \mid n_{2}, n_{3}\right)+q_{1}^{-}\left(n_{1}+1, n_{2}, n_{3}\right) T\left(n_{1}+1 \mid n_{2}, n_{3}\right) \\
& -\left[q_{1}^{+}\left(n_{1}, n_{2}, n_{3}\right)+q_{1}^{-}\left(n_{1}, n_{2}, n_{3}\right)\right] T\left(n_{1} \mid n_{2}, n_{3}\right) .
\end{aligned}
$$

Due to detailed balance, the approximate conditional probabilities $T\left(n_{1} \mid n_{2}, n_{3}\right)$ satisfy the one-term recurrence relation

$$
\begin{aligned}
& K\left[\mu+r^{+}\left(n_{1}-1\right)\right] T\left(n_{1}-1 \mid n_{2}, n_{3}\right)= \\
& \quad n_{1}\left[K r^{-}+m_{1}+r \rho\left(n_{2}+n_{3}\right)\right] T\left(n_{1} \mid n_{2}, n_{3}\right) .
\end{aligned}
$$

Repeating for $S=3$ the procedure devised to estimate the coordinates of the critical points leads to the set of quadratic equations

$n_{1}^{2}+\rho n_{1}\left(n_{2}+n_{3}\right)-K n_{1}+K \alpha=0$,
$n_{2}^{2}+\rho n_{2}\left(n_{1}+n_{3}\right)-K n_{2}+K \alpha=0$,
$n_{3}^{2}+\rho n_{3}\left(n_{1}+n_{2}\right)-K n_{3}+K \alpha=0$,

which yields 8 interior critical points, 6 of which are saddle points, and the remaining two points are a local minimum and, as in $S=2$, a point that is a maximum or a saddle point depending on $\rho$. Explicit expressions for their coordinates are given below. For the sake of comparison we have also calculated critical points using the exact joint distribution $P\left(n_{1}, n_{2}, n_{3}\right)$, see Fig. $1 \mathbf{b}$. 
The coordinates for the interior critical points are: on the one hand, $M_{1}=\left(m_{+}, m_{+}, m_{+}\right)$and $M_{2}=\left(m_{-}, m_{-}, m_{-}\right)$, where

$m_{ \pm}=\frac{K}{2\left(1+2 \beta^{\prime}\right)}\left(1 \pm \sqrt{1-\frac{4 \alpha(1+2 \beta)}{K}}\right)$

Both solutions turn out to be complex when

$\rho>\frac{1}{2}\left(\frac{K}{4 \alpha}-1\right)$

On the other hand, six interior critical points appear at points $Q_{i}$, $i=1, \ldots, 6$, where $Q_{1}=\left(t_{+}, t_{+}, s_{-}\right)$and $Q_{2}, Q_{3}$ are obtained as the cyclic permutations of the entries of $Q_{1}$, whereas $Q_{4}=\left(t_{-}, t_{-}, s_{+}\right)$and the entries of $Q_{5}$ and $Q_{6}$ are the cyclic permutations of that of $Q_{4}$, with

$t_{ \pm}=\frac{K}{2\left(1+\rho^{\prime}\right)}\left(1 \pm \sqrt{1-\frac{4 \alpha\left(1+\rho^{2}\right)}{K\left(1-\rho^{\prime}\right)}}\right)$.

$s_{ \pm}=\frac{K}{2}\left(1 \pm \sqrt{1-\frac{4 \alpha(1+\rho)}{K(1-\rho)}}\right)$.

Both $s_{ \pm}$and $t_{ \pm}$are real whenever $\rho^{p} \rho_{\mathrm{c}}$, where

$P_{\mathrm{c}}=\frac{1-4\left(r^{+}-\mu\right) / K r}{1+4\left(r^{+}-\mu\right) / K r}$.

In spite that Eqs. (17) and (19) have been obtained using an approximate form for conditional probabilities, the numerical calculation of the critical points using the actual distribution is in very good agreement with these approximations (see Fig. 1b). For $\rho<p_{0}, M_{1}$ is classified as a local maximum. At $\rho=\rho_{c}$ all saddle points and $M_{1}$ coalesce in a single point. For $\beta>\beta$, however, $M_{1}$ becomes a saddle point, as can be checked numerically with the Hessian matrix.

Boundary maxima are of the form $(n, n, 0),(n, 0,0)$-and their cyclic permutations. The non-zero coordinates of the former are equal to that of the coexistence maximum $M_{1}$ obtained for 2 species, see Eq. (10); the non-zero entries of the latter are the same as the boundary maxima for $S=2$, see Eq. (13).

\subsubsection{Configuration-state partitioning}

For $S=2$ potential species, a simple way to divide the configuration space is to use saddle points. Fig. 2 depicts steady-state distributions for increasing competitive overlap as well as the location of saddle points. A natural partitioning should relate configurations around the coexistence maximum to species coexistence, and states near the boundary maxima to configurations close to one-species extinction. As Fig. 2 shows, saddle points discriminate with accuracy the configurations that can be associated to coexistence from those that can be related to one-species extinction. According to the coordinate $\left(s_{-}\right)$that closest to the boundary (cf. Eq. (11)), the partitioning results as:
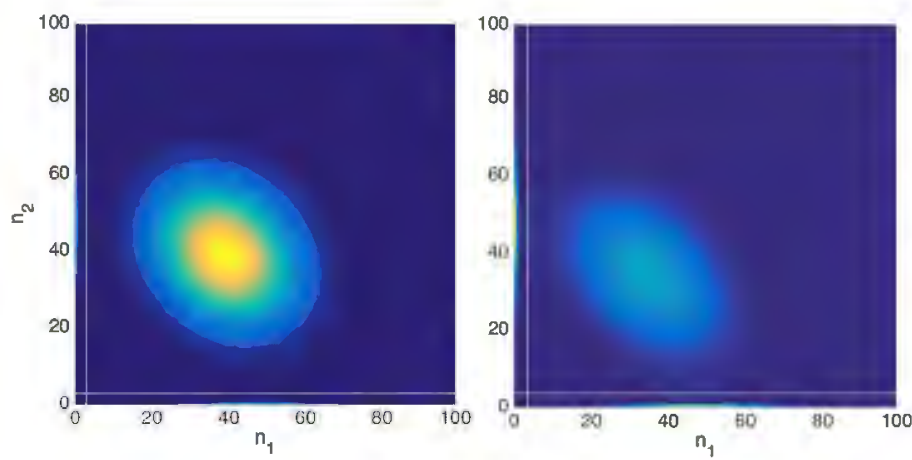

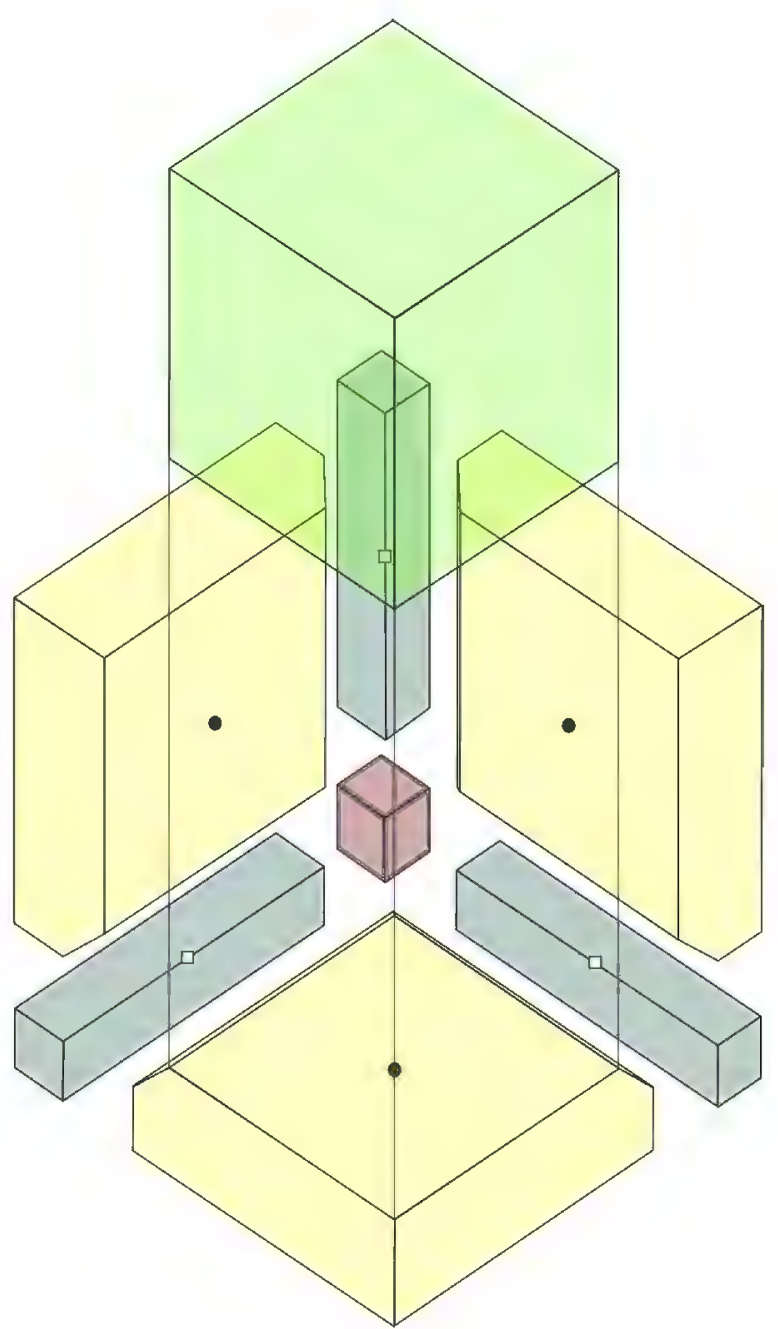

Fig. 3. Partition of the configuration space $\mathbb{N}^{3}$ for $S=3$ potential species. To ease visualization, we have separated $\mathrm{N}^{3}$ into four regions according to saddle points: black circles denote points $Q_{1}, Q_{2}$ and $Q_{3}$, whereas white squares represent saddle points $Q_{4}$. $Q_{5}$ and $Q_{6}$. The latter are used to define regions for complete (red) and two-species extinctions (blue), and the former determine the coexistence volume (green) and the onespecies extinction region (yellow). The (green) cube has been displaced vertically to facilitate visualization. (For interpretation of the references to color in this figure legend, the reader is referred to the web version of this article.)

1. $0 \leq n_{i}<s_{-}$for $i=1,2$. This square is associated with full extinction.

2. $0 \leq n_{1}<s_{-}, n_{2}>s_{-}$or $0 \leq n_{2}<s_{-}, n_{1}>s_{-}$. These two rectangles are associated with the extinction of one species, since configurations are close to extinction in the form $(n, 0)$ or $(0, n)$.
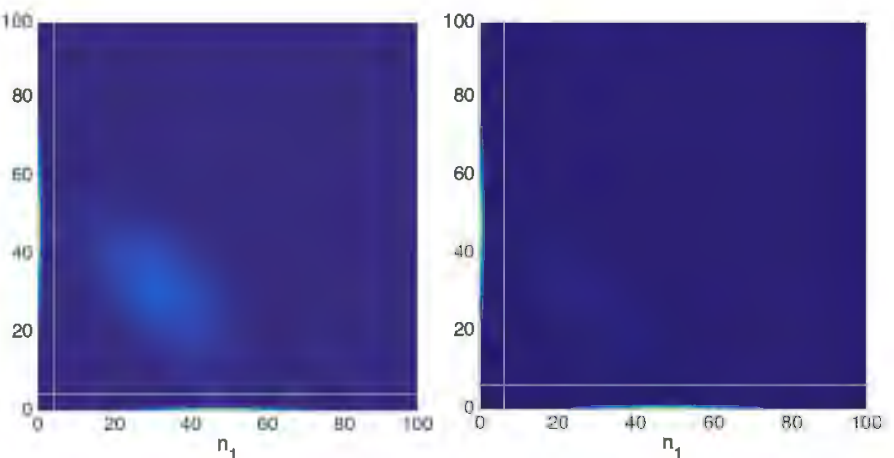

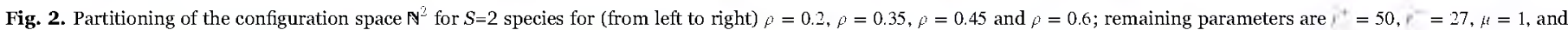

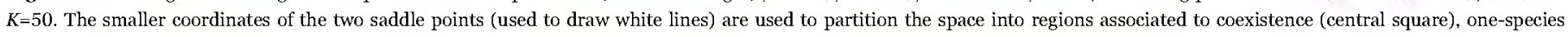

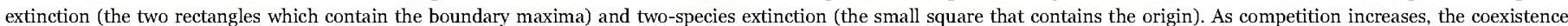
maximum approaches to the origin, and saddle points become closer to the maximum. 
3. $n_{i}>s_{-}$for $i=1,2$. The rest of the configuration space puts together states that can be associated to coexistence.

Note that, strictly speaking, there will be configurations where both $n_{1}, n_{2}>0$ are classified as one- or two-species extinction states. The classification here is meant to separate configurations that are close to boundary maxima in which one or two species are extinct from those that can be associated to coexistence, in which species populations are far from being extinct.

The partitioning for three-species communities simply generalizes the $S=2$ case. Again, each saddle point has a coordinate close to the boundary (cf. $s_{-}$and $t_{-}$in Eq. (19)). Note also that $s_{-}>t_{-}$. Taking this fact into account, saddle points divide the configuration space $\mathbb{N}^{3}$ into several regions, which have been depicted in Fig. 3:

1. $0 \leq n_{i}<t_{-}$for $i=1,2,3$. This cube is associated with the extinction of the three species (since the origin belongs to this region).

2. $0 \leq n_{i}<t_{-}$for $i=1,2$ and $n_{3}>t_{-}$(and the two remaining combinations). These three parallelepipeds are associated with the extinction of two species, because boundary maxima of the form $(n, 0,0)-$ and its cyclic permutations - are situated inside this volume, as well as other population configurations close to two-species extinctions.

3. $n_{i}>s_{-}$for $\dot{i}=1,2,3$. This cube contains the interior maximum and is therefore associated with the coexistence of the three species.

4. The remaining volume of the configuration space, where boundary maxima of the form $(n, n, 0)$-and its cyclic permutations- are located, is associated with the extinction of one species.

The configuration-state partitioning slightly differs from the general case when saddle points have coalesced. If $\rho>\rho_{c}$, the point $M_{1}=\left(m_{+}, m_{+}, m_{+}\right)$is classified as the only saddle point. Based on its coordinates, the configuration space is partitioned as follows:

1. $0 \leq n_{i}<m_{+}$for $i=1,2,3$. This cube is associated with full extinction.

2. $0 \leq n_{i}<m_{+}$for $i=1,2$ and $n_{3}>m_{+}$(and the two remaining combinations). These three parallelepipeds are associated with the extinction of two species.

3. $n_{i}>m_{+}$for $i=1,2,3$. This cube is associated with coexistence configurations.

4. The remaining volume of the configuration space is related to the extinction of one species.

The same partitioning applies for the $S=2$ case when only a single saddle point remains.

\subsection{Extinction cascade}

Saddle points of the joint probability distribution have allowed us to establish a natural partitioning into regions associated to coexistence (containing the coexistence maximum) and to the extinction on one, two, or three species (containing the corresponding boundary maxima), see Fig. 3. Over these regions we aggregate the joint distribution $P\left(n_{1}, n_{2}, n_{3}\right)$, calculated numerically as described in Appendix $\mathrm{C}$, to define the overall probability of coexistence,

$P_{\mathrm{c}}=\sum_{n_{1}, n_{2}, n_{3}>s_{-}} P\left(n_{1}, n_{2}, n_{3}\right)$,

the probability of three-extinct species configurations,

$P_{3}=\sum_{n_{1}, n_{2}, n_{3}<t_{-}} P\left(n_{1}, n_{2}, n_{3}\right)$,

and the probability of two-extinct species, which by symmetry over cyclic permutations of $\left(n_{1}, n_{2}, n_{3}\right)$ can be expressed as

$P_{2}=3 \sum_{n_{1}, n_{2}<t_{-}} \sum_{n_{3}>t-} P\left(n_{1}, n_{2}, n_{3}\right)$.
The probability of one-extinct species, $P_{1}$, is obtained from the normalization condition $P_{\mathrm{c}}+P_{1}+P_{2}+P_{3}=1$. Fig. 4 shows these aggregated probabilities as a function of $\rho$ for two sets of model parameters. In the first case, the coexistence probability is almost one for low values of the competitive overlap and, as $\rho$ increases, at some point the probability declines rapidly. At the same time, the probabilities of one and two extinct species begin to increase. Note that, once the threshold $\rho_{c}$ has been crossed over, the most probable state consists of a single, extant species, and the probability of coexistence becomes negligible.

In the second case, corresponding to a larger value of the mortality rate $r^{-}$, the threshold $\rho_{\mathrm{c}}$ at which the coexistence maximum $M_{1}$ transforms into a saddle point becomes smaller. The probability of coexistence rapidly declines as $\rho$ increases and, in addition, there is a nonnegligible probability of complete extinction. Remarkably, Fig. 4b shows that, for smaller values of the carrying capacity, coexistence is not the only possible state even at $\rho=0$. This puts a practical limit to the maximum number of coexisting species which does have a deterministic counterpart -recall that, due to global stability, the deterministic model permits the packing of an arbitrary number of species for $\rho<1$.

Contrary to the deterministic prediction that $S-1$ extinctions take place abruptly as $\rho$ increases (Appendix B), Fig. 4 shows that ecological drift induces a sequential cascade of extinctions, in which states with a larger number of extinct species are more prone to be observed as the competitive overlap increases.

An important remark is on purpose here. The cascade of extinction we have just described has nothing to do with the degree of synchronicity in which extinctions take place along time, i.e., the term "cascade" does not refer here to a sequential extinction in time. In particular, the symmetric, deterministic model leads in general to asynchronous extinctions. The stochastic phenomenon analyzed in this contribution refers to the progressive extinctions that occur as competitive strength increases.

\subsection{Limit of small demographic stochasticity}

In the absence of stochasticity, the deterministic model predicts the extinction of $S-1$ species once the threshold in competition $\rho=1$ is crossed over. In the stochastic case, the extinction threshold is pushed to smaller values of competitive strength (Capitán et al., 2015), and the probabilities of configurations with one or more extinct species are non-zero in overlapping windows of competition. These two scenarios only differ on the presence or absence of demographic stochasticity, but lead to significantly different outcomes. Therefore, incorporating demographic stochasticity appears to be very relevant in the dynamics of ecological community models.

In order to evaluate the importance of demographic stochasticity, we have tried to quantify the difference between these two scenarios as stochasticity decreases. To do so, we have studied the limit of small stochasticity, in which the deterministic model is to be recovered. As shown below, the transition to the small-stochasticity scenario is abrupt, hence incorporating demographic stochasticity to community models should be strongly considered.

Since fluctuations are expected to decrease as population size increases (see Appendix D), the small-stochasticity limit is equivalent to the limit of large population sizes. In its turn, increasing population sizes is associated to increasing carrying capacity: note that a linear functional dependence between equilibrium densities and carrying capacity is recovered in the deterministic limit $[\widehat{x}=K /(1-\rho+\rho S)$, see Section 2]. In the stochastic case, as confirmed by our analytical approximations for the critical points [cf. Eqs. (10) and (17)], the population sizes for coexistence maxima increase linearly for large $K$, so we have studied the resulting extinction sequence by increasing the carrying capacity at fixed $\rho$. We have quantified the intensity of demographic noise by the coefficient of variation of population abundances, $\nu=\sigma_{n} /\langle n\rangle, \sigma_{n}$ being the standard deviation of population numbers and $\langle n\rangle$ the average value. As shown in Appendix D, when 


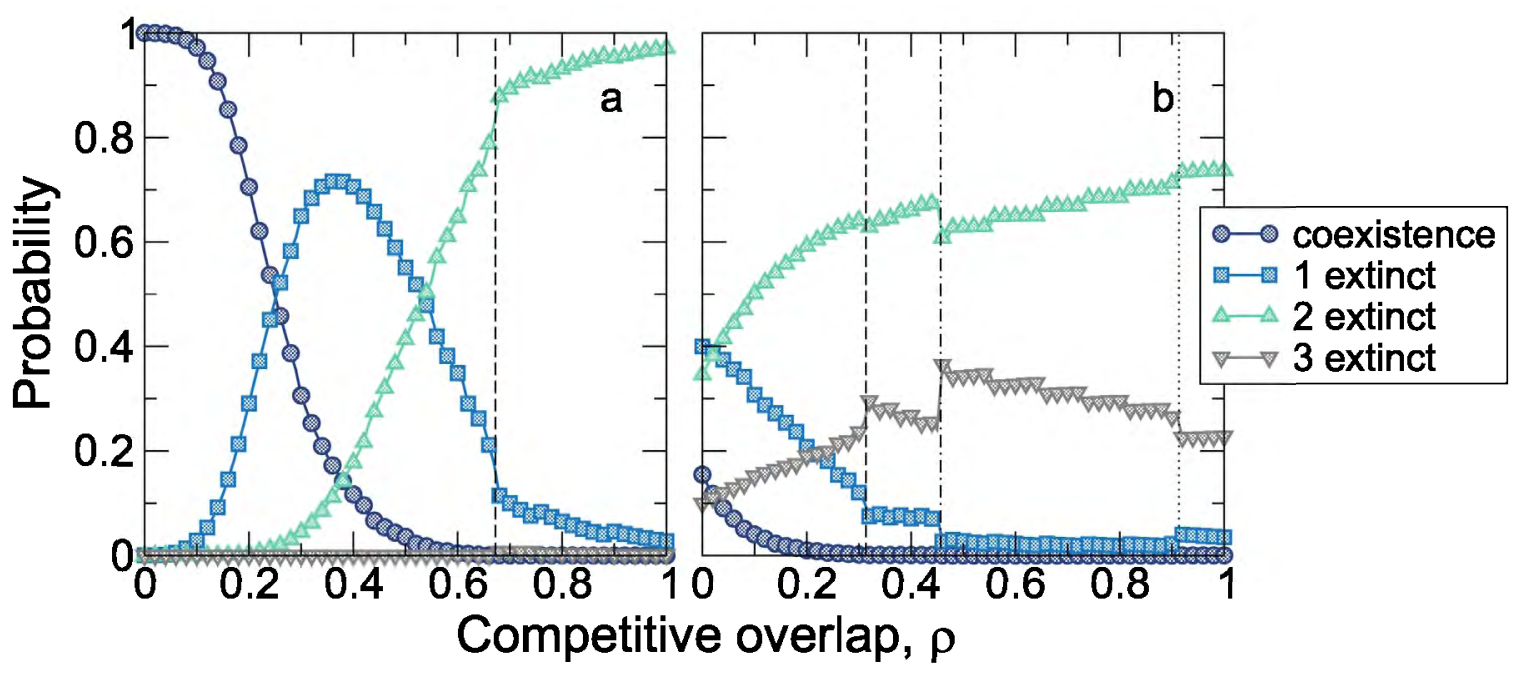

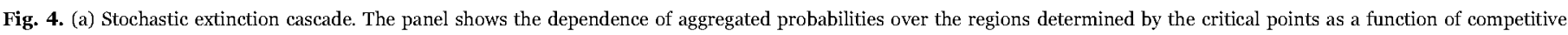

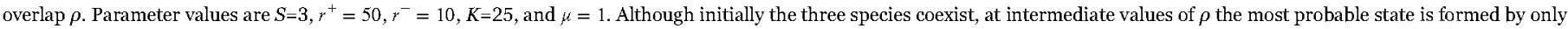

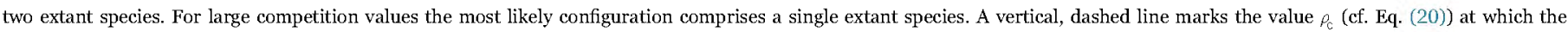

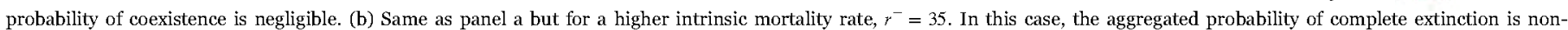

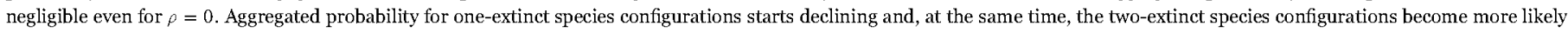

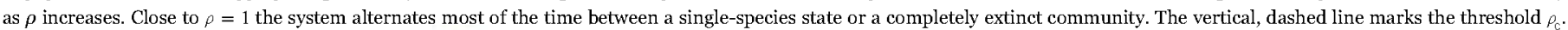

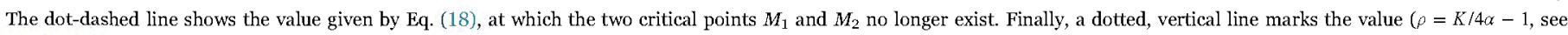
Section 3.1) at which the boundary maxima of the form $(n, n, 0)$-and permutations - no longer exist.

$K \gg 1$ then $\sigma_{n} \sim K^{1 / 2}$ and $\langle n\rangle \sim K$, so $v$ tends to zero in the limit of large population sizes. From the numerical point of view, to get close to the deterministic scenario we would have to choose a carrying capacity value such that the average $\langle n\rangle$ is large enough compared to the variability in populations. In practice, we will assume that the system is close to a low-noise regime when the actual coefficient of variation, obtained though the joint probability distribution of the stochastic model calculated numerically, is close to that obtained by a Gaussian approximation of the joint distribution valid in the limit $K \gg 1$ (see Appendix D). Note that, for the Gaussian approximation to be valid, the coexistence, interior maxima must be located far away from the boundaries, so that the joint probabilities associated to all of extinction states are negligible.

The results of this analysis are summarized in Fig. 5. For low levels of stochasticity, the numerical coefficient of variation and the analytical approximation, Eq. (D.8), remain close to each other. For the corresponding values of $K$, the probability of coexistence is almost equal to one (as in the deterministic scenario). However, as $v$ increases (i.e., the carrying capacity $K$ decreases), the probability that one species becomes extinct grows sharply and, at the same time, the probability of coexistence starts declining. As the variability in population sizes augments, overlapping windows for the likelihood of progressive extinctions arise. The transition to situations where low demographic stochasticity operates is, therefore, abrupt.

Note that the cascade obtained in Fig. $5 \mathrm{~b}$ and $\mathrm{d}$ as a function of noise can be immediately translated into a cascade in carrying capacity (for fixed $\rho$ ). This reinforces our conclusion, since the extinction cascade phenomenon also occurs when other model parameter $(K)$ varies. It is presumably the relative balance between $\rho$ and $K$ that determines the subspace of the parameter space for which extinctions start appearing.

\subsection{Evaluating the role of environmental stochasticity}

In order to test the robustness of our main result against different sources of noise, we have replaced demographic stochasticity by environmental stochasticity. We have introduced variability in model parameters so that, to keep the scenario as simple as possible, the competitive overlap $\rho$ in Eq. (1) is replaced by $\rho+\xi(t)$, where $\xi(t)$ stands for a noise term with zero mean. The deterministic dynamics transforms into a Langevin equation with multiplicative noise,

$\dot{x}_{i}=r x_{i}\left(1-\frac{x_{i}+\rho \sum_{j \neq i} x_{j}}{K}\right)-\frac{r x_{i}}{K}\left(\sum_{j \neq i} x_{j}\right) \xi(t)$,

which can be rewritten as

$z_{i}^{\prime}=z_{i}\left(1-z_{i}-\rho \sum_{j \neq i} z_{j}\right)-z_{i}\left(\sum_{j \neq i} z_{j}\right) \xi(t)$

by re-scaling species densities as $z_{i}=x_{i} / K$ and time as $t^{\prime}=n t$ ( $z_{i}^{\prime}$ stands for the derivative with respect to the scaled time $\left.t^{\prime}\right)$. We choose the noise as $\xi(t)=\rho \kappa \eta_{1}(t)$, where $\eta(t)$ is a Brownian motion. The noise $\xi$ has been scaled by $\rho$ in order to avoid that the overall competitive strength, $\rho+\xi(t)$, becomes negative. Note that the way in which we have introduced environmental stochasticity leads to a density-dependent noise term. Including variability in a density-independent (additive) form does not alter qualitatively our results.

Fig. 6 shows the persistence of the extinction cascade when only environmental stochasticity is considered. This points to the robustness of our results: as for demographic stochasticity, environmental stochasticity also alters the predictions of the deterministic dynamics. Note also that, although the two forms of noise here analyzed are inherently different, they both lead to a cascading phenomenon. It can be shown through the Fokker-Plank approximation of the master equation (Capitán et al., 2015) that demographic stochasticity leads to a square-root multiplicative noise term in the approximate Langevin equation. Environmental noise has been introduced as a (linear) multiplicative noise, though. Despite being different forms of accounting for stochasticity, our main result remains robust and persists in both cases.

There are, however, qualitative differences between the predictions yielded by the model when demographic or environmental stochasticity come into play. First, the range in competition on which the cascade takes place is wider for demographic noise. It seems that, in the presence of environmental noise, the range of the cascade can increase moderately when a larger number of species are to be packed (Fig. 6b). More importantly, a second difference arises: no full extinction is possible in the case of environmental noise. This is a peculiarity, not 

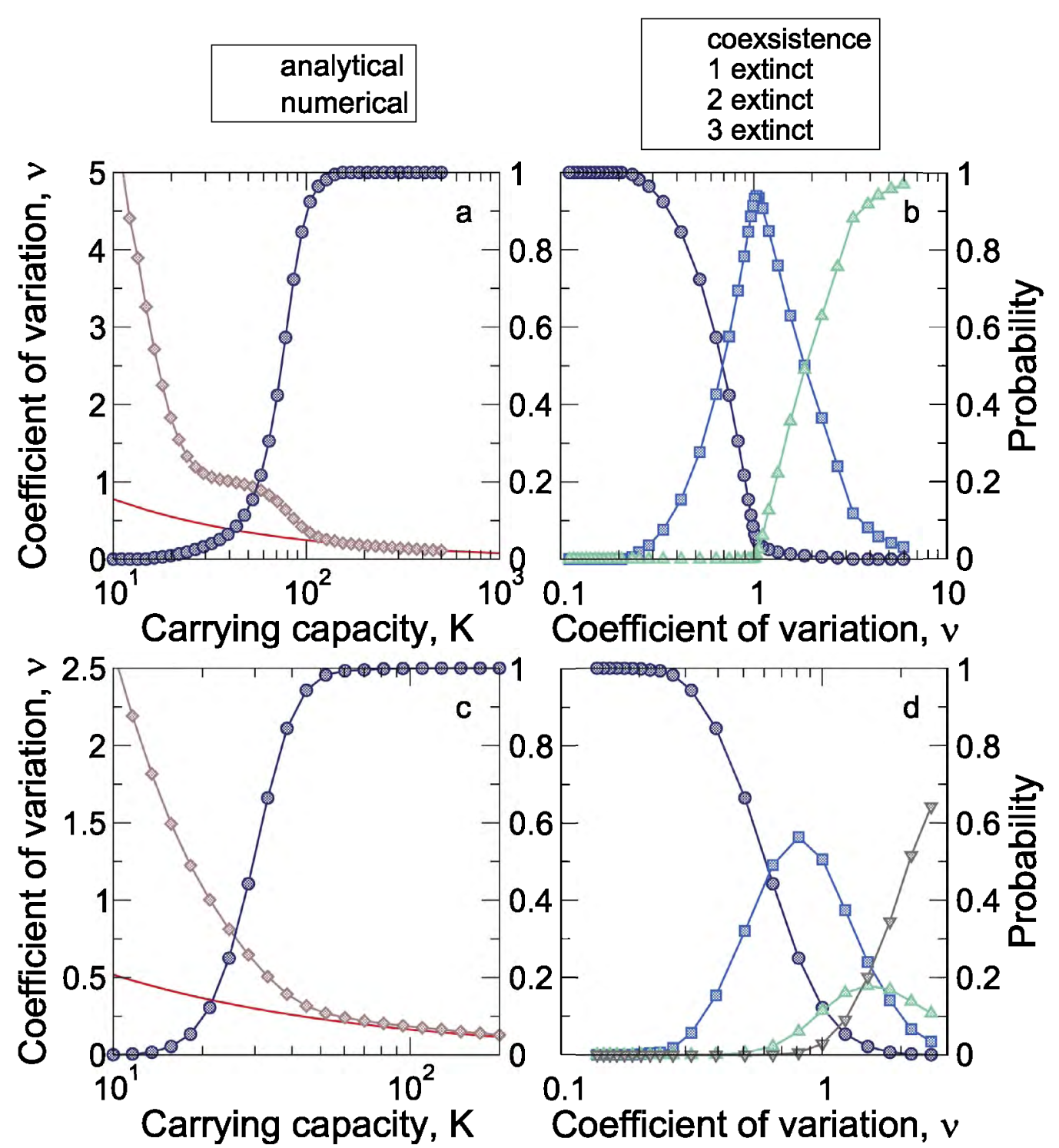

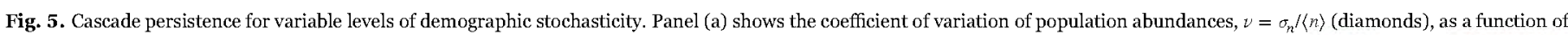

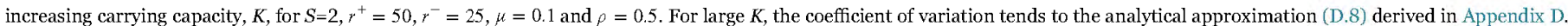

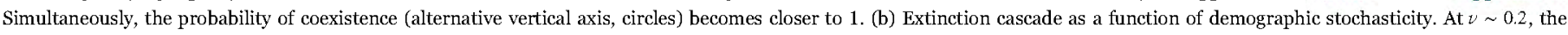

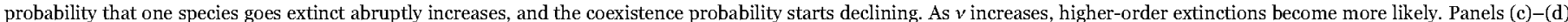

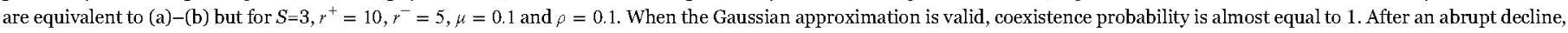
sequential extinctions occur for higher levels of demographic noise.

altered by the noise, of a generic competitive, Lotka-Volterra dynamics (cf. Eq. (A.2) in Appendix A), for which it can be easily shown that the full extinction equilibrium $\left(\widehat{x}_{i}=0, i=1, \ldots, S\right)$ is unstable. The Langevin equation, therefore, can not reproduce configurations associated to the full extinction of the community, contrary to what is observed for demographic stochasticity (Fig. 4b).

\subsection{Relaxing the species symmetry assumption}

In this section we test the robustness of our results by relaxing the assumption of species symmetry in model parameters. It can be argued that the effect of demographic stochasticity simply consists of breaking the symmetry between species. In a generic, non-symmetric, deterministic scenario, one could expect progressive extinctions even in the absence of ecological drift. Therefore, the role of stochasticity would be simply to re-establish a deterministic scenario where one-by-one species extinctions occur. In this subsection we discuss the implications of relaxing the symmetry assumption to determine the true role of demographic stochasticity in a generic case.

In order to address these questions, here we consider two examples of fully non-symmetric, three-species competitive dynamics,

$\dot{x}_{i}=r x_{i}\left(1-\frac{1}{K_{i}} \sum_{j=1}^{3} \rho_{i j} x_{j}\right)$,

where carrying capacities and interspecific competitive strengths are species-dependent. We set, without loss of generality, off-diagonal competition values as $\rho_{12}=\rho_{21}=\rho, \rho_{13}=\rho_{31}=\rho+\delta_{1}, \rho_{23}=\rho_{32}=\rho+\delta_{2}$, and $\rho_{i j}=1$ for $i=1,2,3$.

In the first example we choose $\delta_{1}=0.1, \delta_{2}=0.05, K_{1}=40, K_{2}=16$, and $K_{3}=20$. After analyzing the stability of all the equilibrium points of the deterministic system (see details in Appendix E) we find that, although being fully non-symmetric, this model predicts a two-species, grouped extinction when the threshold $\rho=0.4$ is crossed over (Fig. 7a). For $\rho>0.4$, only equilibria with a single extant species are stable, whereas no other scenario but three-species coexistence is stable for $0 \leq \rho<0.4$. By continuity of the eigenvalues of the Jacobian matrix on 


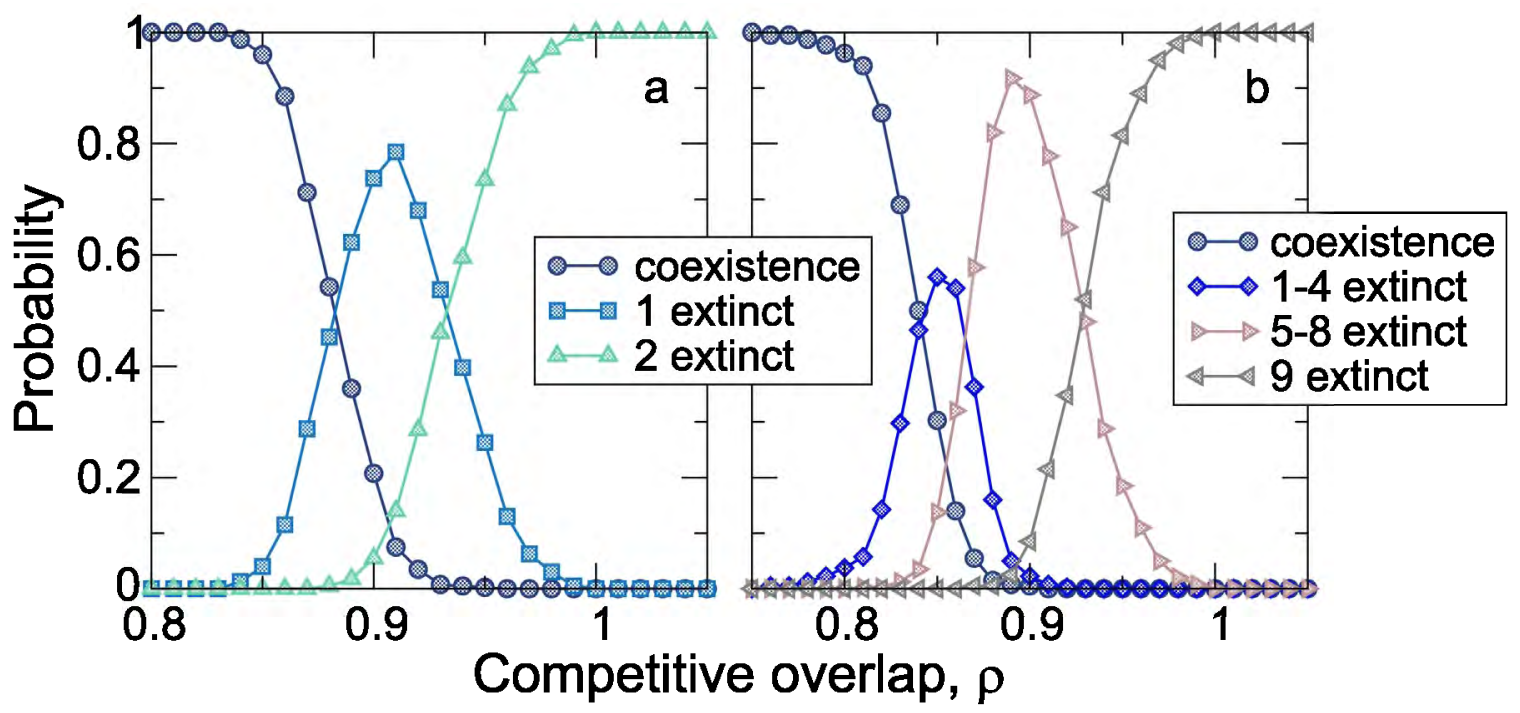

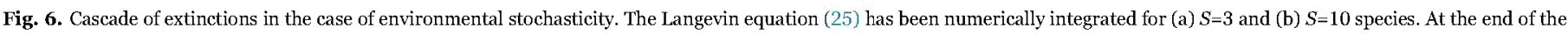

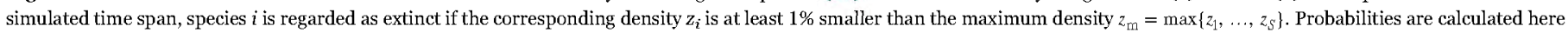

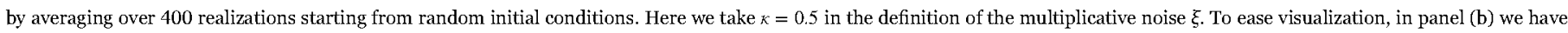

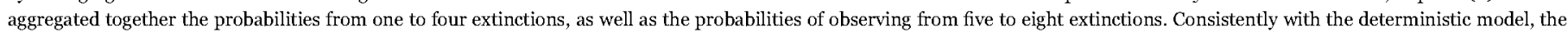
full extinction state is never observed even in the presence of noise.

model parameters, there are multiple non-symmetric, deterministic scenarios that exhibit the transition from full coexistence to a singleextant-species state. Therefore, grouped extinctions are not a peculiarity of the fully symmetric scenario.

In the second example (Fig. 7b), $\delta_{1}=0.4, \delta_{2}=0.3$ and $K_{1}=K_{2}=K_{3}=15$. The complete stability analysis draws the conclusion that non-symmetric models yield to multistability. In Appendix $\mathrm{E}$ it is shown that, for $\rho<0.3$, only the interior equilibrium point is asymptotically stable; for $0.3<\rho<0.7$, only the boundary point $(15 /(1+\rho), 15 /(1+\rho), 0)$ is stable, but for $0.7<\rho<1$, the former boundary point remains stable as well as the single-species extinction equilibrium $(0,0,15)$ (see also Fig. 7b). For $\rho>1$, however, the three boundary equilibria with a single, extant species are the only ones that remain stable. As a consequence, there is a range in competition $(0.7<\rho<1)$ where configurations formed by a single extant species or by two coexisting species co-occur. Depending on the initial condition, the dynamics can end up in one of the two attractors. The basin of attraction of each equilibrium point will determine how frequently one or two species go extinct when competition surpasses the value $\rho=0.7$. This analysis is out of the scope of this contribution, though. Importantly, the extinction sequence in non-symmetric scenarios can depend on initial conditions and is not fully determined in principle.

Multiple extinctions are not precluded in general even when the symmetry between species is broken, as the first example shows. Multistability ranges could also lead to grouped extinctions in deterministic scenarios. In a Lotka-Volterra community model with $S$ species there are $2^{S}$ attractors. Increasing complexity would likely lead to additional overlapping regions in competition where multiple stable attractors co-occur and species can decline together. Moreover, the deterministic extinction can be ambiguous. It seems difficult to establish the conditions under which a general non-symmetric model will produce a well-defined extinction sequence. The variability introduced by idiosyncratic, species-dependent carrying capacities, growth rates, or intra- and interspecific strengths, may cause the extinction

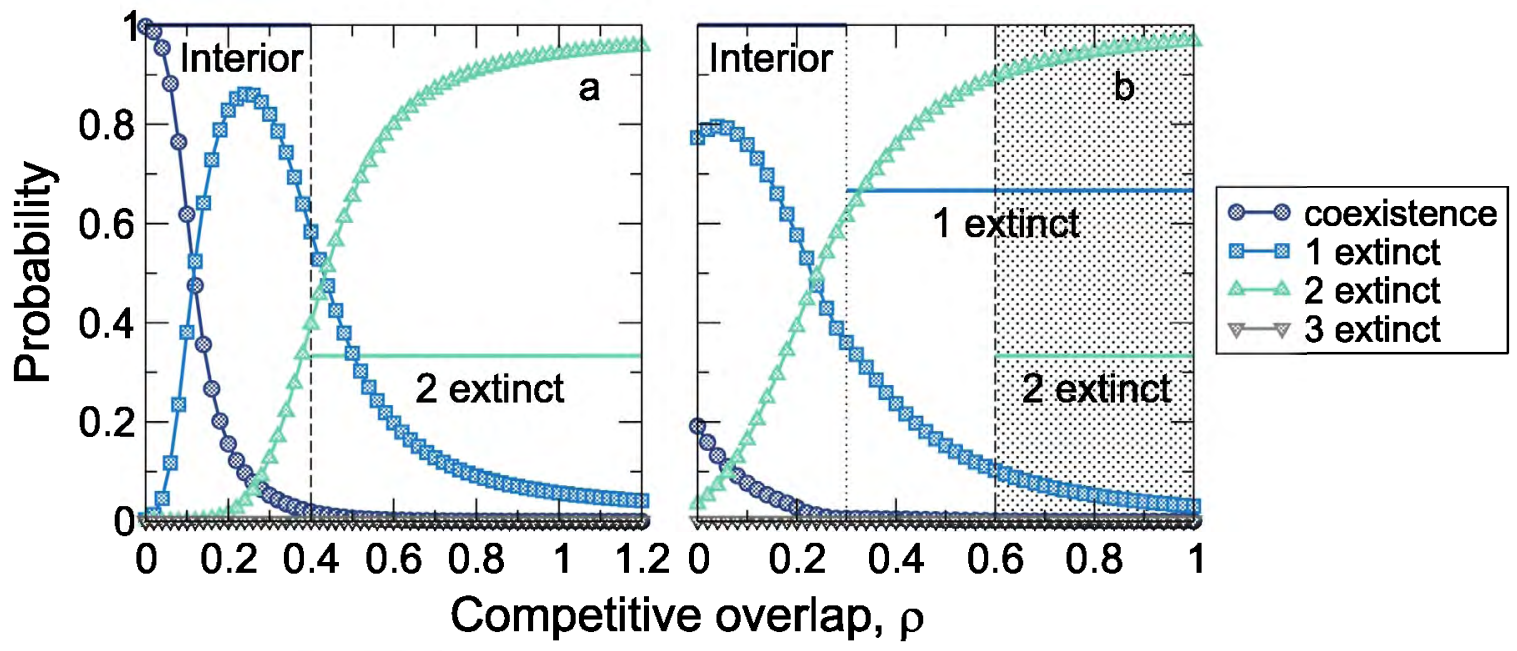

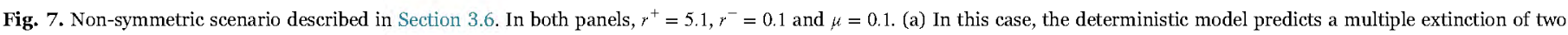

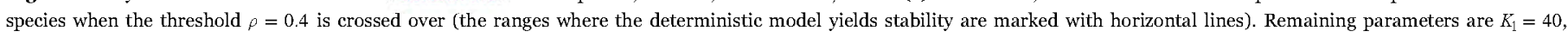

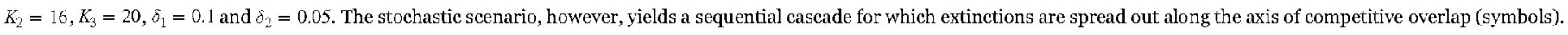

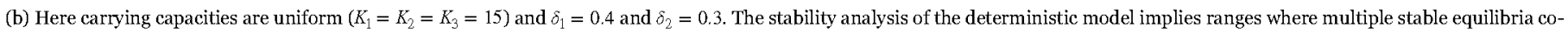
occur (shadowed area). The extinction sequence is not fully determined, and has to be compared with the stochastic prediction. 
sequence to be analytically unpredictable for species-rich communities.

Remarkably, the extinction sequence predicted by non-symmetric, deterministic models has nothing to do with the cascade observed when demographic stochasticity comes into play. We have calculated the probabilities of coexistence and one-, two- or three-species extinctions for both stochastic parametrizations of the two non-symmetric models considered in this subsection. To aggregate joint probabilities, we obtained numerically the critical points by spline interpolation of the exact joint distribution. Now the six saddle points have asymmetric entries, but three of them exhibit a coordinate close to the boundary, and the remaining three saddle points present two coordinates near the axes. Thus, the partition of the configuration space is conceptually equivalent to that of Fig. 3. Not surprisingly, as Fig. 7 evidences, the stochastic model predicts a sequential cascade of extinctions. The threshold at which extinctions start occurring displaces towards smaller values of $\rho$, leading to narrow ranges of effective stochastic coexistence. The most likely state in the presence of stochasticity is not necessarily the same as in a deterministic scenario, the predictions being utterly different in terms of the extinction sequence. Therefore, regardless of the inherent lack of symmetries that deterministic dynamics may have, demographic stochasticity can influence significantly the way in which extinctions take place.

\section{Discussion}

In this contribution, we have analyzed the extinction phenomenon for a symmetric, Lotka-Volterra competitive system formed by $S$ species. In particular, we have focused on the differences between the deterministic system and its stochastic counterpart. Our main result is related to the way in which species extinction proceeds: on the one hand, in the deterministic system, $S-1$ species are driven to extinction at the very point where competitive exclusion starts to operate. On the other hand, we have shown that the overall probabilities of coexistence and one-, two-, or three-species extinction alternate sequentially as the most likely states when competitive overlap increases. Therefore, stochasticity is responsible of a progressive sequence of species extinction, a phenomenon that is absent in the deterministic system. Our analyses are based on a birth-death-immigration stochastic dynamics that was analyzed in deep by Haegeman and Loreau (2011) and later used by Capitán et al. (2015) to unveil the existence of a more restrictive threshold in competition when demographic stochasticity is explicitly considered. In addition to lowering the threshold in competition at which extinctions begin to occur, ecological drift also changes drastically the way those extinctions take place. In order to evidence this difference, we have developed convenient analytical approximations to the critical points of the joint probability for $S=2$ and $S=3$ potential species, which were used to divide the configuration space of the stochastic process to yield aggregated probabilities associated to coexistence and to the corresponding configurations with one or more extinct species. These probabilities reveal the stochastic extinction cascade.

We have shown that different community configurations alternate within certain ranges of competition: coexistence and one-species extinction alternate for small $\rho$, whereas one-species and two-species extinction most likely interchange among each other for intermediate values of $\rho$. The presence of multiple modes in the joint probability distribution is akin to the presence of multistability in a deterministic system. However, the symmetric, deterministic model is characterized by the absence of multiple stable states for $\rho<1$. Hence the extinction cascade described in this work is an entirely new effect caused only by ecological drift. In addition, we have evidenced that the transition to the deterministic model is sharp when the intensity of demographic stochasticity tends to zero. Moreover, we have proven that environmental stochasticity also leads to a cascade of extinctions, although the ranges in competition where extinctions take place are smaller and, more importantly, the full extinction of the community is not possible in this case.

In this work, we have implemented two types of stochasticity: demographic noise (ecological drift) and environmental stochasticity. These are two typical sources of noise that represent, respectively: (i) the variability in discrete population numbers as a consequence of stochastic births and deaths, or (ii) the stochastic variability in model parameters that can be ascribed to changing environmental conditions. Although they are very different implementations of noise, the main result of this manuscript (the stochastic cascade of extinctions) is common to both of them, with some qualitative differences. In the case of demographic stochasticity, we do not impose a particular form for the noise distribution since it directly emerges from the inherent stochastic dynamics of discrete populations whose individuals undergo a number of elementary processes (in principle, the noise distribution would follow from the master equation). Other ways to implement demographic noise have been discussed in the literature (Bonachela et al., 2012), and they would plausibly lead to mechanisms similar to those found here.

It can be argued that the role of stochasticity in the fully symmetric system reduces to break species symmetry and yield to progressive species extinctions, a scenario that can arise in non-symmetric, deterministic approaches. We have illustrated with examples that grouped extinctions are not exclusive of a fully symmetric situation. Moreover, predicting the extinction sequence in non-symmetric, deterministic cases is difficult because multiple stable equilibria can cooccur in ranges of competition. Besides, although the extinction sequence were completely determined, the cascade in the presence of stochasticity can be totally different from that predicted by the deterministic model. We believe that the examples analyzed in this contribution show up the key role of stochasticity in community assembly.

It would be interesting to empirically test the stochastic extinction cascade phenomenon. In principle, a plausible way to conduct the experiment would involve simple protist microcosms where species compete for a shared resource (see, for example, Violle et al. (2010) and references therein). Lowering the amount of resource could be associated to a decrease in the carrying capacity, and we have shown that the extinction cascade mechanism is expected to arise as long as the carrying capacity (resource availability) is reduced, see Fig. 5. For the experiment to reproduce model settings, an individual (immigrant) coming from the species pool should be inserted in the experimental community at certain times. From a time series listing species identities at certain sampling times, one could estimate the probabilities for coexistence and for the extinction of one or more species, and test whether extinctions in empirical systems tend to proceed sequentially or not.

Our work has two important implications: first, we have developed analytical approximations for conditional probabilities in the cases $S=2$ and $S=3$. As we have shown, these functions work well at least around the critical points of the joint probability. Presumably, the techniques proposed here might be extended to approximate the joint probability distribution itself. This approach, however, has to be performed carefully. The simplest way to approximate the three-species joint distribution according to our methodology is to set $P\left(n_{1}, n_{2}, n_{3}\right) \approx T\left(n_{1} \mid n_{2}, n_{3}\right) T\left(n_{2} \mid n_{3}\right) P\left(n_{3}\right)$, where $P\left(n_{3}\right)$ is the marginal, one-species probability distribution, which can be expressed analytically in terms of hypergeometric functions (Haegeman and Loreau, 2011). Apparently, the approximated distribution lacks of an important property of the exact joint distribution: it is not conserved under cyclic permutations of its arguments. Therefore, it is necessary to devise appropriate combinations of conditional probabilities that preserve the symmetry of the joint distribution under cyclic permutations of its arguments. This research direction, together with a generalization for communities formed by more than three species, could be worth pursuing and compared with other approximations to the joint probability, such as those developed by Haegeman and Loreau (2011). 
The second implication of this contribution is that we remark the importance of explicitly considering ecological drift in theoretical frameworks in community ecology. Natural processes are intrinsically stochastic, because changes in population numbers are discrete, so ecological communities are more reliably modeled using stochastic community models, even at regimes where their deterministic limits are not expected to fail. In certain situations, the use of deterministic dynamics in community ecology could lead to utterly different predictions, as we have shown. Traditionally ecology has relied on these kind of models, and currently considerable theoretical progress is made on the basis of deterministic approaches. However, ecological drift may play a determinant role that could not be captured by deterministic formulations. We hope that the present work can inspire new con- tributions in the future that highlight the distinct role of ecological drift in species community models.

\section{Acknowledgements}

We thank the constructive criticisms and comments of the Editor and two anonymous reviewers, who helped to improve significantly the original manuscript. This work was funded by projects SITES (CGL2012-39964, DA, JAC) and BRIDGES (CGL2015-69043-P, DA, JAC) and the Ramón y Cajal Fellowship program (RYC-2010-06545, DA). JAC also acknowledges partial financial support from the Department of Applied Mathematics (Technical University of Madrid).

\section{Appendix A. Deterministic competitive exclusion}

Gause's competitive exclusion principle is usually stated as "two species competing for a single resource cannot coexist". Strictly speaking, the competitive exclusion principle was first formulated by Volterra (1926) as a mathematical proposition. Albeit a mathematical proof of the principle, based on dynamical systems theory, can be found in the book by Hofbauer and Sigmund (1998), and is essentially the same as that of Volterra (1926) original paper, we here provide a purely algebraic alternative demonstration (see Roughgarden (1979) as well).

Assume that the densities of $S$ species living on $R$ resources vary in time according to the dynamics

$\dot{x}_{i}=x_{i}\left(\sum_{j=1}^{R} \gamma_{i j} y_{j}-d_{i}\right), \quad i=1, \ldots, S$,

where $\Gamma=\left(\gamma_{i j}\right)$ is a $S \times R$ matrix with non-negative entries, $y_{j}, j=1, \ldots, R$, are amounts of $R$ resources, which are also assumed to depend on species densities, and $d_{i}, i=1, \ldots, S$ are the rates of decline of species when all resources are zero. We now show that if $S>R$ and species densities reach a well-defined steady state, then in the long run $S-R$ species will go extinct until the number of species equals the number of resources.

Imposing the condition $\dot{x}_{i}=0$ for large $t$ and assuming that all species densities are positive, we get the non-homogeneous linear system $\Gamma \mathbf{y}=\mathbf{d}$, with $\mathbf{y}=\left(y_{i}\right) \in \mathbb{R}^{R}$, and $\mathbf{d}=\left(d_{i}\right) \in \mathbb{R}^{S}$. Given that matrix $\Gamma$ has $S$ rows and only $R<S$ columns, the system will be incompatible except for some specific vectors $\mathbf{d}$ belonging to the image $\operatorname{Im}(T)$ of matrix $\Gamma$. As a consequence, to find an equilibrium solution some species densities must go to zero. For those displaced species, the corresponding rows of matrix $\Gamma$ can be removed until $\operatorname{rank}(T)$ rows remain. If the rank equals the number $R$ of resources, the system turns out to be compatible and determinate, and $R$ species will stably coexist. Note that it is the rank of matrix $I$ rather than the number of resources itself that induces competitive exclusion.

Therefore, competition for shared resources imposes a limit to the maximum number of species that can stably coexist. This result has a counterpart in the stability of Lotka-Volterra equations derived from the MacArthur's consumer-resource model (MacArthur, 1970; Chesson, 1990), i.e., the particular case of Eq. (A.1) in which per-capita resource growth rates $y_{i} / y_{i}$ depend linearly on population densities. In the limit of fast resource variation, the dynamics (A.1) takes the Lotka-Volterra form (Chesson, 1990),

$\dot{x}_{i}=r_{i} x_{i}\left(1-\frac{x_{i}+\sum_{j \neq i} \rho_{i j} x_{j}}{K_{i}}\right), \quad i=1, \ldots, S$.

Here $r_{i}$ is interpreted as the intrinsic growth rate of species $i, K_{i}$ as a carrying capacity, and $\rho_{i j}$ measures interspecific competition strength between species $i$ and $j$ relative to intraspecific competition. As shown by Chesson (1990), if $S=R=\operatorname{rank}(T)$, then exists a unique solution of the system $x_{i}+\sum_{i \neq i} \rho_{i j} x_{j}=K_{i}, i=1, \ldots, S$. The resulting equilibrium point will be interior if every species density remains strictly positive. Moreover, Chesson (1990) demonstrated that if there exists a unique interior equilibrium point for the dynamics (A.2), it will be globally stable if and only if $S=R=\operatorname{rank}(T)$. Therefore, if competitive exclusion does not operate, a unique globally stable equilibrium point is reached and, conversely, if the Lotka-Volterra equations present an interior, globally stable equilibrium point, any positive initial condition will make the dynamics (A.2) converge to the equilibrium point. This automatically ensures that none of the $S$ species is driven to extinction by competitive exclusion (MacArthur, 1970 ; Takeuchi, 1996).

\section{Appendix B. Stability of the symmetric, deterministic model for $\rho \geq 1$}

In this Appendix we perform a stability analysis of the equilibrium points of the symmetric, deterministic dynamics in the competition regime $\rho \geq 1$. As we mentioned before, when $\rho<1$ it can be shown that the interior equilibrium point is globally stable (Hofbauer and Sigmund, 1998 ; Capitán et al., 2015), all boundary equilibria being unstable.

We start by analyzing stability for $\rho=1$. In this case, the system is also stable and the initial condition determines the attractor which the dynamics converges to. Any equilibrium point is such that its densities satisfy

$\sum_{j=1}^{S} x_{j}=K$.

Let $J(t)=\sum_{j=1}^{S} x_{j}(t)$. Then, by summing up the equations of the system (1) for $\rho=1$ we get

$\frac{d J}{d t}=r J\left(1-\frac{J}{K}\right)$ 
which can be integrated and yields

$J(t)=\left[\frac{1}{K}+\left(\frac{1}{J_{0}}-\frac{1}{K}\right) e^{-r t}\right]^{-1}$,

$J_{0}$ being the initial condition for $J(t), J_{0}=J(0)=\sum_{j=1}^{S} x_{j}(0)$. Therefore, the dynamics of each species turns out to be decoupled,

$\frac{d x_{i}}{d t}=r x_{i}\left(1-\frac{J(t)}{K}\right)$

The equilibrium point to which (B.4) converges is determined by the initial condition vector $\mathbf{x}_{0}=\left(x_{1}(0), \ldots, x_{S}(0)\right)$. For two distinct species $i$ and $j$, (B.4) implies that $\dot{x}_{i} / \dot{x}_{j}=x_{i} / x_{j}$. Integration yields $x_{i}(t) / x_{j}(t)=x_{i}(0) / x_{j}(0)$, which means that the proportions of population densities are conserved along the dynamics, whose orbits are reduced to straight lines starting from the initial condition $\mathbf{x}_{\mathbf{0}}$ along the direction determined by the vector $\mathbf{x}_{0}$. Therefore, the final equilibrium point is given by the intersection of the hyperplane $\sum_{j=1}^{S} x_{j}=K$ and the line that links the initial condition point, $\mathbf{x}_{0}$, and the origin. Any of these (infinite) equilibrium points will be stable, provided that the initial densities satisfy $x_{i}(0) \geq 0$ for all $i$.

The case $\rho>1$ leads to competitive exclusion. We analyze the asymptotic stability of the $2^{S}$ equilibrium points with positive or zero densities. Without loss of generality, for $0 \leq n \leq S$ any equilibrium point will be of the form

$\mathbf{x}_{n}=\left(\begin{array}{c}\widehat{x}_{n} \mathbf{u}_{S-n} \\ \mathbf{0}_{n}\end{array}\right)$,

where subscript $n$ indicates that $n$ densities are strictly equal to 0 , and $\mathbf{u}_{n}=(1, \ldots, 1)^{\mathrm{T}}$ is a vector with $n$ entries equal to 1 . Any equilibrium with $n$ zero densities can be written as a permutation of (B.5), without altering the subsequent stability analysis. In addition, the non-zero entries of $\mathbf{x}_{n}$ are the solutions of the system

$(1-\rho) x_{i}+\rho \sum_{j=1}^{S-n} x_{j}=K, \quad i=1, \ldots, S-n$.

This system admits a single solution for which the $S-n$ species have equal densities, $x_{i}=\widehat{x}_{n}$, where

$\widehat{x}_{n}=\frac{K}{1-\rho+\rho(S-n)}$.

Our demonstration reduces to show that, if $\rho>1$, all the non-trivial equilibria act as repellors except for $n=1$, i.e., when only a single species survives. To this purpose we evaluate the eigenvalue spectra of the Jacobian matrix of the system (1). The Jacobian matrix $\mathbf{J}$ can be expressed in a block form as

$\mathbf{J}=\left(\begin{array}{ll}\mathrm{J}_{11} & \mathbf{J}_{12} \\ \mathrm{~J}_{21} & \mathbf{J}_{22}\end{array}\right)$

where

$\mathbf{J}_{11}=-\frac{r \widehat{x}_{n}}{K}\left[(1-\rho) \mathbf{I}_{S-n}+\rho \mathbf{u}_{S-n} \mathbf{u}_{S-n}^{\mathrm{T}}\right]$,

$\mathbf{J}_{12}=-\frac{r \rho \widehat{x}_{n}}{K} \mathbf{u}_{S-n} \mathbf{u}_{n}^{\mathrm{T}}$,

$\mathbf{J}_{21}=\mathbf{0}_{n} \mathbf{0}_{S-n}^{\mathrm{T}}$,

$\mathbf{J}_{22}=\frac{r(1-\rho) \widehat{x}_{n}}{K} \mathrm{I}_{n}$,

and $\mathrm{I}_{n}$ is the $n \times n$ identity matrix and $\mathbf{0}_{n}=(0, \ldots, 0)^{\mathrm{T}}$ is the zero vector with $n$ entries. Without loss of generality, eigenvectors can be written as

$\mathbf{v}=\left(\begin{array}{c}\mathbf{a}_{S-n} \\ \mathbf{b}_{n}\end{array}\right)$

where $\mathbf{a}_{S-n}$ and $\mathbf{b}_{n}$ are column vectors with dimensions $S-n$ and $n$, respectively. First let us assume that $\mathbf{b}_{n}=\mathbf{0}_{n}$. The spectral problem reduces to $\mathbf{J}_{11} \mathbf{a}_{S-n}=\lambda \mathbf{a}_{S-n}$.

Two different solutions arise: if $\mathbf{u}_{S-n}^{\mathrm{T}} \mathbf{a}_{S-n}=0$, i.e., $\mathbf{a}_{S-n}$ is orthogonal to $\mathbf{u}_{S-n}$, then the eigenvalue is

$\lambda=-\frac{r(1-\rho) \widehat{x}_{n}}{K}$

with algebraic multiplicity $S-n-1$. However, if $\mathbf{a}_{S-n} \propto \mathbf{u}_{S-n}$ we find $\lambda=-r$ as an eigenvalue with algebraic multiplicity equal to 1 .

On the other hand, if $\mathbf{b}_{n} \neq \mathbf{0}_{n}$ we have to solve

$\mathbf{J}_{11^{1}} \mathbf{a}_{S-n}+\mathbf{J}_{12} \mathbf{b}_{n}=\lambda \mathbf{a}_{S-n}, \mathbf{J}_{22} \mathbf{b}_{n}=\lambda \mathbf{b}_{n}$

Since $\mathrm{J}_{22}$ is proportional to $\mathrm{I}_{n}$, we find

$\lambda=\frac{r(1-\rho) \widehat{x}_{n}}{K}$,

with $n$ eigenvectors $\mathbf{b}_{n}=\mathbf{e}_{n}^{(i)}(i=1, \ldots, n), \mathbf{e}_{n}^{(i)}$ being the $i$-th vector of the canonical basis of $\mathbb{R}^{n}$. The algebraic multiplicity associated to (B.17) is equal 
to $n$. Substituting these results into the first equation of (B.16) yields

$\left[(1-\rho) \mathbf{I}_{S-n}+\rho \mathbf{u}_{S-n} \mathbf{u}_{S-n}^{\mathrm{T}}\right] \mathbf{a}_{S-n}+\rho \mathbf{u}_{S-n}=-(1-\rho) \mathbf{a}_{S-n}$.

Given the structure of the matrices involved, we look for a solution of the form $\mathbf{a}_{S-n}=\alpha \mathbf{u}_{S-n}$, which implies a non-trivial value $\alpha=-\rho /[2(1-\rho)+\rho S]$.

Two eigenvalues determine the asymptotic stability of all the equilibrium points: $\lambda_{1}=-r(1-\rho) \widehat{x}_{n} / K$ and $\lambda_{2}=-\lambda_{1}=r(1-\rho) \widehat{x}_{n} / K$ (the third eigenvalue, $\lambda_{3}=-r$, is always negative). If $\rho>1$ and $0 \leq n<S-1, \lambda_{1}>0$ and remains as an eigenvalue -recall that its multiplicity is $S-n-1>0$. Therefore, any equilibrium with less than $S-1$ extinct species is asymptotically unstable -including the interior coexistence equilibrium. However, when only one species survives $(n=S-1), \lambda_{1}$ is not an eigenvalue anymore and the two other eigenvalues remain: $\lambda_{2}=r(1-\rho) \widehat{x}_{n} / K<0$ (with multiplicity $\left.S-1\right)$ and $\lambda_{3}=-r<0$ (with multiplicity 1 ). Thus, only the $S$ boundary equilibria with a single extant species are asymptotically stable.

Since the trivial equilibrium point (associated to complete extinction) is obviously unstable, we deduce that any orbit starting from an interior initial condition will be repelled if it gets close to any equilibrium, except when the equilibrium point is formed by a single extant species. If the orbit enters the basin of attraction of any of those $S$ equilibria, it will end up in it asymptotically. This implies the extinction at a time of $S-1$ species if $\rho>1$.

\section{Appendix C. Numerical calculation of the steady-state probability distribution}

In order to compute numerically the stationary joint distribution, we limit the infinite configuration space to the set $\Xi \equiv\left\{0,1, \ldots, n_{\max }\right\}^{s}$ by choosing $n_{\max }$ large enough so that the probability of finding a population number equal to $n_{\max }$ is negligible (we choose $n_{\max }$ as the integer part of $2 K$, which fulfills the requirement).

The stationary distribution is obtained by solving the embedded Markov chain associated to the continuous-time Markov process (Karlin and Taylor, 1975). The transition matrix of the embedded Markov chain is defined by the transition probabilities

$\operatorname{Pr}\left\{\mathbf{n} \rightarrow \mathbf{n} \pm \mathbf{e}_{i}\right\}=\frac{q_{i}^{ \pm}(\mathbf{n})}{\Lambda(\mathbf{n})}$,

where $\Lambda(\mathbf{n})=\sum_{i=1}^{S}\left[q_{i}^{+}(\mathbf{n})+q_{i}^{-}(\mathbf{n})\right]$. The remaining transitions have zero probability. Elementary events (overall births and deaths) take place after exponential times, so that the time lapsed to the next event is drawn from a random variable $\tau$ with cumulative distribution $\operatorname{Pr}(\tau \leq t)=1-e^{-\Lambda(\mathbf{n}) t}$. Once the steady-state distribution $\varphi=(\varphi(\mathbf{n}))$ of the embedded Markov chain -i.e., the left-eigenvector of the transition matrix with eigenvalue $1-$ has been determined, according to the mean time spent by the process at state $\mathbf{n}$, the probability of finding the continuous-time Markov process at state $\mathbf{n}$ turns out to be (Cinlar, 1975)

$P(\mathbf{n})=\frac{\varphi(\mathbf{n}) \Lambda(\mathbf{n})^{-1}}{\sum_{\mathbf{m} \in \Xi} \varphi(\mathbf{m}) \Lambda(\mathbf{m})^{-1}}$

\section{Appendix D. Coefficient of variation of population abundances in the limit of large carrying capacity}

In this section we derive an analytical expression for the coefficient of variation of population abundances in the small stochasticity limit. In the case of demographic stochasticity, low variability levels can be obtained for large population sizes or, equivalently, in the limit of large carrying capacity. We build on the Gaussian approximation for the joint probability distribution, which is valid in the limit $K \gg 1$ since the probability of extinction configurations is expected to be negligible, and can be fully calculated for a generic community of size $S$.

The Gaussian approximation can be obtained as the solution to the Fokker-Planck equation deduced from the master Eq. (2). We do not reproduce its derivation here; it can be found at the Supplemental Information of Capitán et al. (2015). Under this approximation, the joint probability distribution is expressed as

$\Pi(\mathbf{n})=\frac{1}{Z} \exp \left\{-\left(\mathbf{n}-\hat{x} \mathbf{u}_{S}\right)^{\mathrm{T}} \mathrm{Q}\left(\mathbf{n}-\hat{x} \mathbf{u}_{S}\right)\right\}$,

where $Z$ is an appropriate normalization factor,

$\hat{x}=\frac{K}{2(1-\rho+\rho S)}\left[1+\sqrt{1+\frac{4 \mu(1-\rho+\rho S)}{r K}}\right]$,

and the covariance matrix $\mathrm{Q}^{-1}$ is given by

$\mathrm{Q}^{-1}=\frac{b}{2 a}\left(\mathrm{I}_{S}-\frac{c}{a+c S} \mathbf{u}_{S} \mathbf{u}_{S}^{\mathrm{T}}\right)$.

In terms of model parameters, $a=r \widehat{x}(1-\rho) / K+\mu \hat{x}, b=2\left(r^{+} \hat{x}+\mu\right)$, and $c=r \rho \widehat{x} / K$. In the large carrying capacity limit, the average population abundance is expressed through a series expansion on powers of $K$ as

$\langle n\rangle=\widehat{x}=\frac{K}{1-\rho+\rho S}+\frac{\mu}{r}+O\left(K^{-1}\right)$.

Similarly, series expansions give

$a=\frac{r(1-\rho)}{1-\rho+\rho S}+O\left(K^{-1}\right), b=\frac{2 r^{+} K}{1-\rho+\rho S}+2 \mu\left(\frac{r^{+}}{r}+1\right)+O\left(K^{-1}\right), c=\frac{r \rho}{1-\rho+\rho S}+O\left(K^{-1}\right)$.

Inserting these expressions into (D.3) yields, up to order $K^{0}$, the approximation 
$\mathrm{Q}^{-1}=\frac{r^{+} K}{r(1-\rho)}\left(\mathrm{I}_{S}-\frac{\rho}{1-\rho+\rho S} \mathbf{u}_{S} \mathbf{u}_{S}^{\mathrm{T}}\right)+O\left(K^{0}\right)$

Therefore the standard deviation of population abundance, $\sigma_{n}$, can be obtained as the square root of diagonal elements of matrix $\mathrm{Q}^{-1}$,

$\sigma_{n}=\sqrt{\frac{r^{+}(1-2 \rho+\rho S)}{r(1-\rho)(1-\rho+\rho S)}} K^{1 / 2}+O\left(K^{-1 / 2}\right)$

which (not surprisingly) scales with $K$ as $K^{1 / 2}$. Finally, the coefficient of variation of population abundances is expressed, in the limit $K \gg 1$, as

$\nu=\frac{\sigma_{n}}{\langle n\rangle}=\sqrt{\frac{r^{+}(1-2 \rho+\rho S)(1-\rho+\rho S)}{r(1-\rho)}} K^{-1 / 2}$.

Strictly speaking, the deterministic scenario $(\nu=0)$ is only achieved in the limit $K \rightarrow \infty$. However, low stochasticity regimes can be assessed using Eq. (D.8): if the actual coefficient of variation is close to that yielded by the Gaussian approximation, both of which are small for large $K$, then extinction configurations are precluded and the variability of populations with respect to the mean value is small. We adopt, as a practical definition for low stochasticity, the parameter combinations for which the actual coefficient of variation is close to the approximation given by Eq. (D.8).

\section{Appendix E. Stability analysis for two deterministic models with non-symmetric competition}

Here we analyze the stability of the equilibrium points of the two non-symmetric, three-species competitive dynamics of the form (26) considered in the main text, for which the interaction matrix is written as

$\mathbf{R}=\left(\rho_{i j}\right)=\left(\begin{array}{ccc}1 & \rho & \rho+\delta_{1} \\ \rho & 1 & \rho+\delta_{2} \\ \rho+\delta_{1} & \rho+\delta_{2} & 1\end{array}\right)$

$\delta_{1}$ and $\delta_{2}$ being two positive numbers. The sign of equilibrium densities and the corresponding eigenvalues of the Jacobian matrix determine the ranges of $\rho$ for which the system is stable. We impose the condition $\rho \geq 0$ for all interaction coefficients to remain positive. Since the growth rate $r>0$, in both cases the full extinction equilibrium $\hat{\mathbf{x}}=(0,0,0)$ is unstable.

In the first example, $\delta_{1}=0.1$ and $\delta_{2}=0.05$. Species carrying capacities are non-uniform: $K_{1}=40, K_{2}=16$, and $K_{3}=20$. Although the expressions are too cumbersome to be reproduced here, it can be shown that the interior equilibrium point $\hat{\mathbf{x}}=\mathbf{R}^{-1} \mathbf{K}$, with $\mathbf{K}=\left(K_{1}, K_{2}, K_{3}\right)^{\mathrm{T}}$, has three positive densities and is asymptotically stable if and only if $0 \leq \rho<0.4$.

We now consider all boundary equilibria. In what follows the eigenvalues $\lambda$ of the stability (Jacobian) matrix are expressed as $\lambda=\lambda^{\prime} r$, i.e., they are scaled by the growth rate $r>0$ :

(a) The first and second coordinates of

$\widehat{\mathbf{x}}=\left(\frac{8(5-2 \rho)}{1-\rho^{2}}, \frac{8(2-5 \rho)}{1-\rho^{2}}, 0\right)$

are positive if and only if $0 \leq \rho<0.4$ or $\rho>2.5$. The scaled eigenvalues $\lambda^{\prime}$ are given by

$\lambda^{\prime} \in\left\{-1, \frac{38-131 \rho+90 \rho^{2}}{50\left(1-\rho^{2}\right)},-\frac{(5-2 \rho)(2-5 \rho)}{10\left(1-\rho^{2}\right)}\right\}$.

The condition $\lambda_{i}^{\prime}<0$ for $i=1,2,3$ yields $1.055<\rho<2.5$. Therefore, this point is never feasible and stable at the same time.

(b) For

$\widehat{\mathbf{x}}=\left(\frac{200(19-10 \rho)}{99-20 \rho-100 \rho^{2}}, 0, \frac{800(2-5 \rho)}{99-20 \rho-100 \rho^{2}}\right)$,

we require positivity for the first and third entries, which yields $0 \leq \rho<0.4$ or $\rho>1.9$. On the other hand, the eigenvalues in this case are

$\lambda^{\prime} \in\left\{-1, \frac{94-345 \rho-275 \rho^{2}}{(9-10 \rho)(11+10 \rho)},-\frac{2(2-5 \rho)(19-10 \rho)}{(9-10 \rho)(11+10 \rho)}\right\}$.

Stability implies $0.9<\rho<1.9$, which is incompatible with the feasibility condition.

(c) The third equilibrium with a single extinct species is

$\widehat{\mathbf{x}}=\left(0, \frac{2000(3-4 \rho)}{399-40 \rho-400 \rho^{2}}, \frac{1280(6-5 \rho)}{399-40 \rho-400 \rho^{2}}\right)$.

This point is feasible if and only if $0 \leq \rho<0.75$ or $\rho>1.2$. The (scaled) eigenvalues of the Jacobian matrix are

$\lambda^{\prime} \in\left\{-1, \frac{-20(3-4 \rho)(6-5 \rho)}{(19-20 \rho)(21+20 \rho)}, \frac{1899-1830 \rho-200 \rho^{2}}{(19-20 \rho)(21+20 \rho)}\right\}$.

Since the system of inequalities $\lambda_{i}^{\prime}<0(i=1,2,3)$ turns out to be incompatible, this point is unstable for all values of $\rho$.

(d) $\hat{\mathbf{x}}=(40,0,0)$ : the scaled eigenvalues are $\{-1,(2-5 \rho) / 2,2(2-5 \rho) / 5\}$. This point is stable for $\rho>0.4$.

(e) $\hat{\mathbf{x}}=(0,16,0): \lambda^{\prime} \in\{-1,(5-2 \rho) / 5,4(6-5 \rho) / 25\}$. The equilibrium point is stable if and only if $\rho>2.5$.

(f) $\widehat{\mathbf{x}}=(0,0,20): \lambda^{\prime} \in\{-1,(19-10 \rho) / 20,5(3-4 \rho) / 16\}$. Asymptotic stability is achieved for $\rho>1.9$. 
As a result, in the range $0 \leq p<0.4$, the only stable point is the coexistence equilibrium. However, for $\beta>0.4$, only two-extinct species equilibria remain asymptotically stable. Since the eigenvalues are continuous functions of model parameters, close to this example we can find multiple nonsymmetric systems that exhibit a grouped, two-species extinction as $\rho$ increases.

The second example shows that multiple stable equilibria can co-occur when interactions are chosen non-symmetrically. In this case, we have taken $\delta_{1}=0.4, \delta_{2}=0.3$ and $K_{1}=K_{2}=K_{3}=15$. The densities of the interior equilibrium point are expressed as

$x_{1}=15(9-10 p)(7-10 p) / D(p), x_{2}=30(3-5 p)(11-10 p) / D(p), x_{3}=150(3-10 p)(1-p) / D(p)$.

where $D(\rho)=75-116 \rho-160 \rho^{2}+200 \rho^{3}$. The feasibility analysis of the equilibrium point yields, for $\rho \geq 0$, the ranges $0 \leq \beta<0.3$ or $0.7<\rho<0.9$ or $\rho>1$.1. The eigenvalues of the stability matrix can be fully calculated, although their expressions are too cumbersome to be reproduced here. It is easy to check that the three eigenvalues are negative if and only if $0 \leq p<0.3$. Therefore, this equilibrium point is interior and asymptotically stable if and only if $0 \leq p<0.3$.

We now summarize the stability analysis for boundary equilibria. All of them are feasible, so stability is only conditioned by the sign of eigenvalues (which are all real):

(a) $\widehat{\mathbf{x}}=\left(\frac{15}{1+\rho}, \frac{15}{1+\rho}, 0\right)$ : the scaled eigenvalues $\lambda^{\prime}$ are $\left\{-1, \frac{3-10 \rho}{10(1+\rho)}, \frac{-1+\rho}{1+\rho}\right\}$. Therefore, this point is stable for $0.3<\rho<1$.

(b) $\hat{\mathbf{x}}=\left(\frac{75}{7+5 p}, 0, \frac{75}{7+5 p}\right): \lambda^{\prime} \in\left\{-1, \frac{11-10_{p}}{2\left(7+5 p^{p}\right.}, \frac{-3+5 p}{7+5 p}\right\}$. The stability conditions form an unfeasible problem, so this point turns out to be unstable for any $\rho$.

(c) $\hat{\mathbf{x}}=\left(0, \frac{150}{13+10 p}, \frac{150}{13+10_{p}}\right): \lambda^{\prime} \in\left\{-1, \frac{9-10 p}{13+10_{p}}, \frac{-7+10 p}{13+10_{p}}\right\}$. Again, this point is unstable for all values of competitive overlap.

(d) $\hat{\mathbf{x}}=(15,0,0)$ : the scaled eigenvalues are $\left\{-1, \frac{3}{5}-\rho, 1-\rho\right\}$. This point is stable for $\rho>1$.

(e) $\hat{\mathbf{x}}=(0,15,0): \lambda^{\prime} \in\left\{-1, \frac{7-10}{10}, 1-p\right\}$. The equilibrium point is stable if and only if $\rho>1$.

(f) $\widehat{\mathbf{x}}=(0,0,15): \lambda^{\prime} \in\left\{-1, \frac{3-5 \beta}{5}, \frac{7-10 y}{10}\right\}$. Stability is attained for $\rho>0.7$.

Consequently, in the range $0.3<p<0.7$ the only stable equilibrium point is $\left(\frac{15}{1+p}, \frac{15}{1+p}, 0\right)$. However, for $0.7<p<1$ two stable equilibria co-occur: the former and a two-extinct species equilibrium, $(0,0,15)$. Depending on initial conditions, the dynamics can lead to one of them or to the other. For $\rho>1$, however, the three equilibria with a single extant species are the only ones that remain asymptotically stable.

This example shows how the cascade of extinctions in non-symmetric, deterministic models can be far from being determined due to the cooccurrence of multiple stable equilibria.

\section{References}

Alonso, D., McKane, A.J., Pascual, M., 2007. Stochastic amplification in epidemics. J. R. Soc. Interface 4, 575-582.

Alonso, D., Ostling, A., Etienne, R.S., 2008. The implicit assumption of symmetry and the species abundance distribution. Ecol. Lett. 11, 93-105.

Black, A.J., McKane, A.J., 2012. Stochastic formulation of ecological models and their applications. Trends Ecol. Evol. 27, 337-345.

Bolker, B.M., Grenfell, B.T., 1995. Space, persistence, and dynamics of measles epidemics. Proceedings R. Soc. Lond. B 348, 308-320.

Bonachela, J.A., Muñoz, M.A., Levin, S.A., 2012. Patchiness and demographic noise in three ecological examples. J. Stat. Phys. 148, 723-739.

Capitán, J.A., Cuesta, J.A., 2011. Species assembly in model ecosystems I: analysis of the population model and the invasion dynamics. J. Theor. Biol. 269, 330-343.

Capitán, J.A., Cuesta, J.A., Bascompte, J., 2009. Statistical mechanics of ecosystem assembly. Phys. Rev. Lett. 103, (168101-4).

Capitán, J.A., Cuenda, S., Alonso, D., 2015. How similar can co-occurring species be in the presence of competition and ecological drift? J. R. Soc. Interface 12, 20150604.

Capitán, J.A., Cuesta, J.A., Bascompte, J., 2011. Species assembly in model ecosystems II: results of the assembly process. J. Theor. Biol. 269, 344-355.

Chesson, P.L., 1990. MacArthur's consumer-resource model. Theor. Popul. Biol. 37, 26-38.

Cinlar, E., 1975. Introduction to Stochastic Processes. Prentice-Hall, New York.

Gause, G.F., 1934. The Struggle for Existence. Williams \& Wilkins, Baltimore, MD.

Haegeman, B., Loreau, M., 2011. A mathematical synthesis of niche and neutral theories in community ecology. J. Theor. Biol. 4, 263-271.

Haigh, J., Maynard-Smith, J., 1972. Can there be more predators than prey? Theor. Popul. Biol. 3, 290-299.

Hardin, G., 1960. The competitive exclusion principle. Science 131, 1292-1297.

Hotbauer, J., Sigmund, K., 1998. The Theory of Evolution and Dynamical Systems. Cambridge. University Press, Cambridge.

Hubbell, S.P., 2001. The Unified Theory of Biodiversity and Biogeography. Princeton University Press, Princeton.

Karlin, S., Taylor, H.M., 1975. A First Course in Stochastic Processes. Acadenic Press, New York.

Law, R., Morton, R.D., 1993. Alternative permanent states of ecological communities. Ecology $74,1347-1361$

Law, R., Morton, R.D., 1996. Permanence and the assembly of ecological communities. Ecology $77,762-775$.

Levin, S.A., 1970. Community equilibria and stability, and an extension of the competitive exclusion principle. Am. Nat. 104, 413-423.

MacArthur, R.H., Levins, R., 1967. The limiting sinilarity, convergence and divergence of coexisting species. Am. Nat. 101, 377-385.

MacArthur, R.H., 1968. Population biology and evolution. In: Lewontin, R.C. (Ed.), The Theory of the Nirhe Syracuse University Press, Syracuse, 159-176.

MacArthur, R.H., 1969. Species packing and what interspecific competition minimizes. Proc. Nat. Acad. Sci. USA 64, 1369-1371.

MacArthur, R.H., 1970. Species packing and competitive equilibria for many species. Theor. Popul. Biol. 1, 1-11.

May, R.M., MacArthur, R.H., 1972. Niche overlap as a function of environmental variability. Proc. Nat. Acad. Sci. USA 69, 1109-1113.

Pielou, E.C., 1977. The number of trophic levels in ecological communities. Nature 268 $329-331$.

Post, W.M., Pimm, S.L., 1983. Community assembly and food web stability. Math. Biosc. 64 $169-192$.

Rosindell, J., Hubbell, S.P., Etienne, R.S., 2011. The unified neutral theory of biodiversity and biogeography at age ten. Trends Ecol. Evol. 26, 451-457.

Roughgarden, J., 1974. Species packing and the competition function with illustrations form Coral Reef Fish. Theor. Popul. Biol. 5, 163-186.

Roughgarden, J., 1979. Theory of Population Genetics and Evolutionary Ecology: An Introduction. MacMillan Publishing, New York.

Schoener, T.W., 1974a. Alternatives to Lotka-Volterra competition: models of intermediate complexity. Theor. Popul. Biol. 10, 309-333.

Schoener, T.W., 1974b. Resource partitioning in ecological communities. Science 185, 27-39.

Takenchi, Y., 1996. Global Dynanical Properties of Lotka-Volterra Systems. World Scientific, Singapore.

Turelli, M., 1978. A reexamination of stability in randomly varying versus deterministic environments with comments on the stochastic theory of liniting sinilarity. Theor. Popul. Biol. 13, 244-267.

Turelli, M., 1980. Niche overlap and invasion of competitors in random environments II: The effects of demographic stochasticity, In: et al., W.J. (Ed.), Biological growth and spread. Springer-Verlag, Berlin, pp. 119-129.

Turelli, M., 1981. Niche overlap and invasion of competitors in random environments I: models without demographic stochasticity. Theor. Popul. Biol. 20, 1-56.

Vellend, M., 2010. Conceptual synthesis in community ecology. Q. Rev. Biol. 85, 183-206.

Violle, C., Pu, Z., Jiang, L., 2010. Experimental demonstration of the importance of competition under disturbance. Proc. Nat. Acad. Sci. USA 107, 12925-12929.

Volterra, V., 1926. Variazioni e fluttuazioni del numero d'individui in specie animali conviventi. Mem. R. Acad. Naz. dei Lincei 2, 31-113.

Wang, R.H., Jin, Z., Lin, Q.X., van de Koppel, J., Alonso, D., 2012. A simple stochastic model with environmental transmission explains multi-year periodicity in outbreaks of avian flu. PLos One 7 (1-9), e28873

Wisz, M., Pottier, J., Kissling, W.D., Pellisier, L., Lenoir, J., Damgaard, C., Dormann, C., Forchhanmer, M.C., Grytnes, J.A., Guisan, A., Heikkinen, R., Hoye, T.T., Kühn, I., Luoto, M., Maiorano, L., Nilsson, M.C., Normand, S., Öckinger, E., Schmidt, N.M., Ternansen, M., Timmermann, A., Wardle, D., Aastrup, P., Svenning, J.C., 2013. The role of biotic interactions in shaping distributions and realised assemblages of species: implications for species distribution modelling. Biol. Rev. 88, 15-30. 